\title{
Magneto-optical transmission in magnetic nanoparticle suspensions for different optical applications: A review
}

\author{
Dengwei Jing *,a ${ }^{\text {, L Sun }}{ }^{a}$, Jingyu Jin, ${ }^{a}$ Madasamy Thangamuthu, ${ }^{b}$ Junwang Tang *,b \\ ${ }^{a}$ State Key Laboratory of Multiphase Flow in Power Engineering \& International Research Center for \\ Renewable Energy, Xi'an Jiaotong University Xi'an 710049, China \\ ${ }^{b}$ Departement of Chemical Engineering, University College London, Torrington Place, London WC1E 7JE, U.K. \\ * Corresponding author: Email: dwjing@mail.xjtu.edu.cn; Junwang.tang@ucl.ac.uk
}

\begin{abstract}
In recent years, the magneto-optical properties of magnetic nanofluids have received increasing attention due to their wide range of applications in various solar energy conversion processes and also as smart fluids in tunable photonic device, optical switch, optical fiber sensor, etc. This article reviews up-to-date developments in magneto-optical transmission in ferrofluids for different optical applications. With magnetic field, the magnetic nanoparticle will undergo interesting structural transitions including chainlike formation and lateral coalescence, leading to various light transmission phenomena in ferrofluids. The orientation of the magnetic field relative to the incident light has a significant influence on the intensity of transmitted light through the suspensions. For incident light along the magnetic field, the polarization direction of light has a negligible effect on the magneto-optical transmission. However, the polarization direction of light should make a great difference for light normal to the field direction. These recent studies are comprehensively reviewed, and their main findings and potential applications are also presented and discussed. Our study is supposed to provide a general view on the research trends, existing problems and future work for the investigation of magneto-optical transmission in magnetic nanofluids.
\end{abstract}

Key words: Magnetic nanofluids, magneto-optical transmission, transmitted light intensity, particle aggregation

\section{Contents}

1. Introduction 2

2. Field-induced aggregation of magnetic nanoparticles 3

3. Recent studies for magneto-optical transmission 4 
4. Applications

5. Conclusions .23

Acknowledgments

References 24

\section{Introduction}

Magnetic nanofluids (MNFs), also called ferrofluids, are colloidal dispersions containing magnetic nano-sized particles $\left(\mathrm{Fe}_{3} \mathrm{O}_{4}, \gamma-\mathrm{Fe}_{2} \mathrm{O}_{3}\right)$ and base fluids (hydrocarbons, water, and so on). They have both the magnetism similar to other solid magnetic materials and fluidity like other liquids $[1,2]$. When there is no magnetic field, the suspensions are isotropic. Once the external field is applied, they show anisotropic characteristics and exhibit magneto-optic phenomena, for example birefringence, dichroism, magneto-optical transmission, etc [3-6]. In recent years, the magneto-optical properties of ferrofluids have received extensive attention due to their wide range of applications like tunable photonic devices, holographic optical tweezers, sensors and so on [7].

Light transmission in suspensions containing magnetic particles has been a topic of interest both theoretically and experimentally [8-12]. Because of the limitations to prepare monodispersed nanofluids with good stability [13], the early studies mainly focused on ferrofluids composed of micron-sized magnetic particles. The magneto-optical effects in magnetic nanofluids were first explored in 2008 by Philip et al. [13, 14]. They found the transmitted light intensity in ferrofluids would decrease dramatically at a critical magnetic field where the incident light was set to be along the field orientation. The critical field intensity followed a power law decay with the nanoparticle content suggesting the structure transformation. Besides, the transmitted intensity was observed to recover to the initial state when the external field disappeared. Based on careful analysis, they proposed the resonances inside the aggregated nanoparticles should be the origin for such interesting findings. Followed by them, Rablau et al. [15] investigated the time evolution of magnetooptical transmission behaviors in magnetic nanofluids. For light along the field direction, the transmitted intensity was found to decrease rapidly to zero and then it would increase gradually with the time. Moreover, they reported the characteristic time $\tau_{0}$ for the extinction of light varied approximately inversely with the external field intensity. The time 
evolution of light transmission could be rationalized through consideration of particle aggregation processes. Eloi et al. [16] further studied the field dependence of light transmission in ferrofluids made up by nanosized particles. A different theory was proposed by them that the rotating of particle aggregates should result in the reduced light transmission in the initial stage. Moreover, they also concluded that the emergence of columnar aggregates should be responsible for the minimum transmitted intensity.

In 2012, Philip and Laskar [7] reviewed the magneto-optical transmission studies in ferrofluids and their potential applications. After that, the light transmission in ferrofluids was still a hot topic and gained increasing concern in the past several years [17-20]. However, to the best of our knowledge, the recent studies related to magneto-optical transmission in magnetic nanofluids are not reviewed yet. Therefore, the objective of our work is to review the recent developments in magneto-optical transmission in magnetic nanofluids for different optical applications. Since the structural transition has a great influence on the light transmission, the field-induced particle aggregation is thus briefly introduced in Section 2 . Recent researches with respect to magneto-optical transmission in ferrofluids are summarized in Section 3, mainly including two cases that the incident beam is orientated along and normal to the field direction respectively. Besides, recent studies in regard to other orientations between light and magnetic field are discussed as well. In Section 4, some potential applications for the magneto-optical transmission in ferrofluids are presented. Finally, the recent research findings and the future work are concluded in Section 5 .

\section{Field-induced aggregation of magnetic nanoparticles}

The magneto-optical transmission in ferrofluids is closely related to the particle aggregation process. Therefore, to better understand the light transmission behaviors, the field-induced particle aggregation is first introduced in this section.

When there is no magnetic field, nano-sized particles are randomly distributed in ferrofluids due to Brownian motion [21]. Once the external field is switched on, the suspended nanoparticles can acquire dipole moments, which tend to get along with the field direction. The effective magnetic interaction between magnetized particles against their thermal energy can be described by the following parameter $[22,23]$ :

$$
L=\frac{\pi \mu_{0} d^{3} \chi^{2} H^{2}}{72 k_{B} T}
$$

where $\mu_{0}$ represents the permeability of vacuum, $d$ and $\chi$ represent the diameter and the susceptibility of magnetic nanoparticles respectively, $T$ represents the temperature, $k_{\mathrm{B}}$ represents the Boltzmann constant and $H$ represents the field intensity. When the value of $L$ is much larger than 1, the Brownian particles in ferrofluids will self-assemble to form chainlike structures (Fig. 1(a)) that are along the field direction [22, 23]. This aggregation process has been confirmed by experiments [24-26] and numerical simulation [27-29]. 
Under the external field, the particle chains can further coarsen to form column structures (Fig. 1(b)) through lateral coalescence. It was found that, with the increase of field strength, the chain-like clusters would get much longer and much thinker as well [21]. Moreover, the lateral coalescence can also be a time-dependent process dominant by three proposed mechanisms including thermal fluctuation $[30,31]$, defect perturbation [31, 32] and lateral interaction [33]. A HT theory was first proposed by Halsey and Toor [30] that a long-range interaction between particle chains can be generated by the thermal fluctuation, causing the thermally driving coarsening. However, Martin et al. [31, 32] found the lateral coalescence can be induced by topological defect in particle chains without thermal fluctuation. It was also speculated by them that the particle roughness should have a great impact on the aggregation process. A following study reported by Furst and Gast [33] showed that the lateral interaction between individual chains was expected to govern the long-term variation of particle structures in the suspensions. Besides, they observed that rigid chains should interact with each other only at short distances, while flexible chains performed attraction in the long range which agreed well with HT theory.

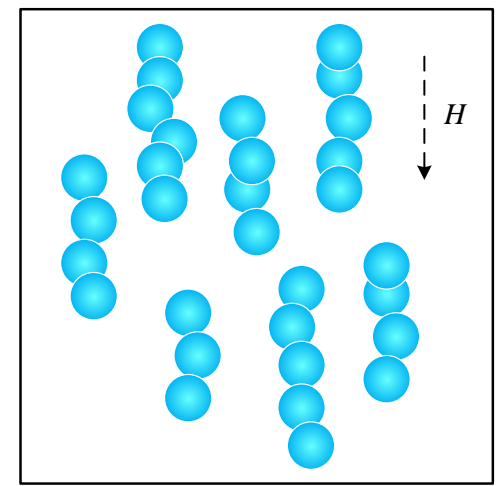

(a)

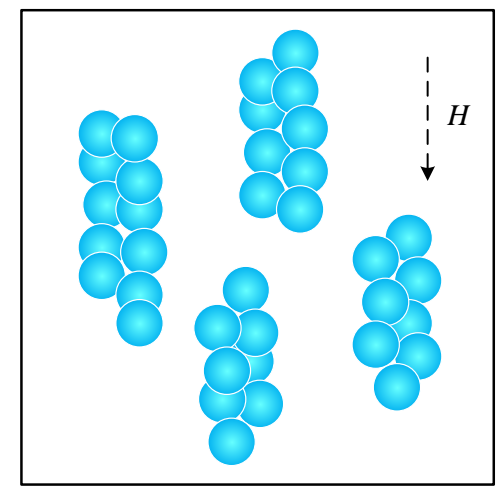

(b)

Fig. 1 Schematic diagram of field-induced particle aggregation in ferrofluids. (a) Chainlike formation. (b) Lateral coalescence.

\section{Recent studies for magneto-optical transmission}

Most recent studies with respect to the magneto-optical transmission in magnetic nanofluids were conducted for the two orientations between incident light and magnetic field: light along and normal to the field direction respectively. These two typical cases are the focus in our study and the relevant literatures are comprehensively reviewed in the following. Besides, several cases for other orientations are also briefly introduced and discussed.

\subsection{Light along the field direction}

Recent studies related to the light along the field direction are presented in this section. It should be noted that Laskar et al. [34] has proved that the light polarization direction has negligible influence on the optical transmission in ferrofluids for light along the magnetic field. Therefore, the effect of light polarization was not considered in the below- mentioned studies. Huang et al. [35] investigated the transmitted light relaxation behaviors in ferrofluids composed of $\mathrm{CoFe}_{2} \mathrm{O}_{4}$ nanoparticles $(\sim 10 \mathrm{~nm})$ under gradient magnetic fields. When a low-gradient magnetic field was applied, it was found that 
the transmitted intensity first reduced to the minimum and then it gradually increased to its initial value. Finally, the intensity either increased or decreased slowly depended on the field gradient direction. This relaxation process was explained by the formation of particle columns and their following motion caused by the external field. Under the highgradient field, they found that the transmitted intensity reduced continuously and showed a slower trend compared to that under the field with low gradient.

Li et al. [36] studied the magneto-optical relaxation behaviors in ferrofluids containing $\gamma-\mathrm{Fe}_{2} \mathrm{O}_{3} / \mathrm{Ni}_{2} \mathrm{O}_{3}$ composite nanoparticles under centripetal gradient magnetic field. The central field intensity is 300 Gs and the field gradient is 30 Gs $\mathrm{mm}^{-1}$. Results show that the transmitted light through $\gamma-\mathrm{Fe}_{2} \mathrm{O}_{3} / \mathrm{Ni}_{2} \mathrm{O}_{3}$ ferrofluid samples performs similar relaxation behavior as other ferrofluids, indicating the formation of particle chains and their following motion. They also investigated the light transmission properties of binary ferrofluids containing ferromagnetic $\gamma-\mathrm{Fe}_{2} \mathrm{O}_{3} / \mathrm{Ni}_{2} \mathrm{O}_{3}(\mathrm{~A}$ particles, $11.21 \pm 0.28$ $\mathrm{nm}$ ) and noncrystalline $\mathrm{Fe}_{2} \mathrm{O}_{3}$ (B particles, $6.54 \pm 0.35 \mathrm{~nm}$ ) nanoparticles in the presence of centripetal gradient magnetic field [17]. Their results indicate that the transmitted light tends to stabilize faster for binary ferrofluids with higher volume fractions of weak magnetic B particles. This phenomenon should be attributed to the variation of the magnetic divergent force as well as the viscosity drag force in the damping-like aggregation process of A-particle chains.

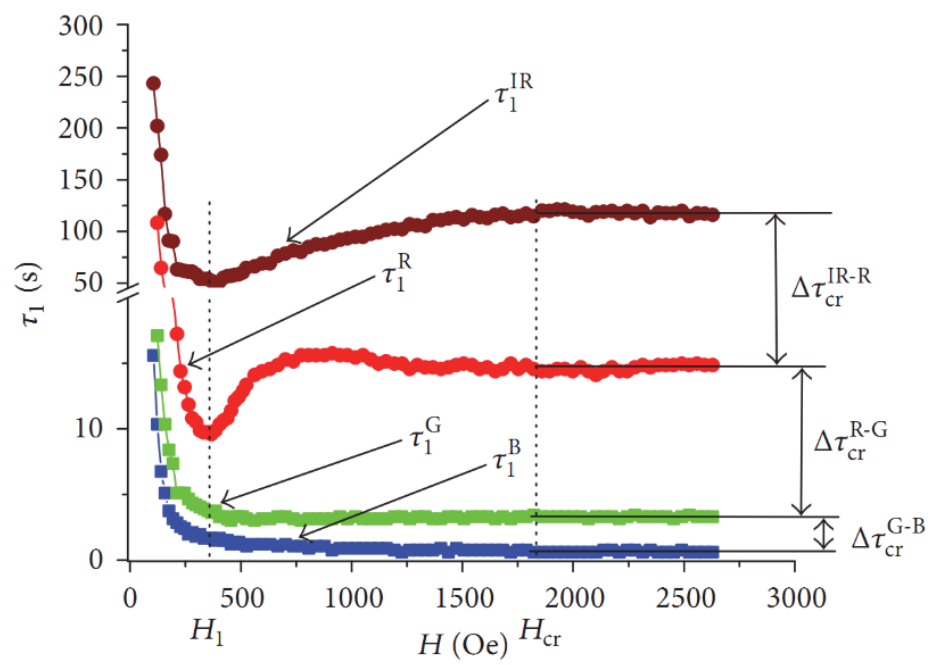

Fig. 2 Magneto-optical extinction trend inversion dependencies of characteristic time $\tau_{1}$ on the magnetic field pulse intensity at incident light wavelengths of $\lambda_{\mathrm{B}}, \lambda_{\mathrm{G}}, \lambda_{\mathrm{R}}$, and $\lambda_{\mathrm{IR}}$, repectively [37].

Optical transmission properties of thin water-based $\mathrm{Fe}_{3} \mathrm{O}_{4}$ ferrofluid layers under pulse uniform magnetic field were experimentally investigated by Shulyma et al. [19]. The nanoparticle has an average diameter of $11.5 \mathrm{~nm}$ and the volume fraction of the suspensions was set to be $1.2 \%$. Results reveal that, under the influence of external field, the transmitted intensity first decreased rapidly and then recovered to its initial state even before the disappearance the field pulse. The observed phenomenon is termed as magneto-optical extinction trend inversion (ETI). The characteristic inversion time $\tau_{1}$ was found to be dependent on both the magnetic pulse intensity and the samples thickness. The experimental outcomes can 
be explained by the growth of columnar particle chains and lateral aggregation, resulting in a transition of light scattering type for Rayleigh scattering to Mie scattering. Moreover, by molecular dynamics simulation, they demonstrated that a uniform magnetic field can give rise to the formation of particle chains and their coalescence in the suspensions, which is quite different from the known Li theory [38]. They further studied the ETI phenomenon in the kerosene-based $\mathrm{Fe}_{3} \mathrm{O}_{4}$ ferrofluid layers at various optical radiation wavelengths, namely, $\lambda_{\mathrm{B}}=450 \pm 10 \mathrm{~nm}, \lambda_{\mathrm{G}}=530 \pm 10 \mathrm{~nm}, \lambda_{\mathrm{R}}=630 \mathrm{~nm}$, and $\lambda_{\mathrm{IR}}=1060 \pm 10 \mathrm{~nm}$ [37]. Results plotted in Fig. 2 indicate the characteristic inversion time $\tau_{1}$ shifts to smaller values at lower incident wavelengths. However, the ETI phenomenon was not observed for very dilute $\left(\varphi_{\mathrm{V}} \leq 0.3 \%\right)$ and very concentrated $\left(\varphi_{\mathrm{V}} \geq 2 \%\right)$ ferrofluid samples.

(a)

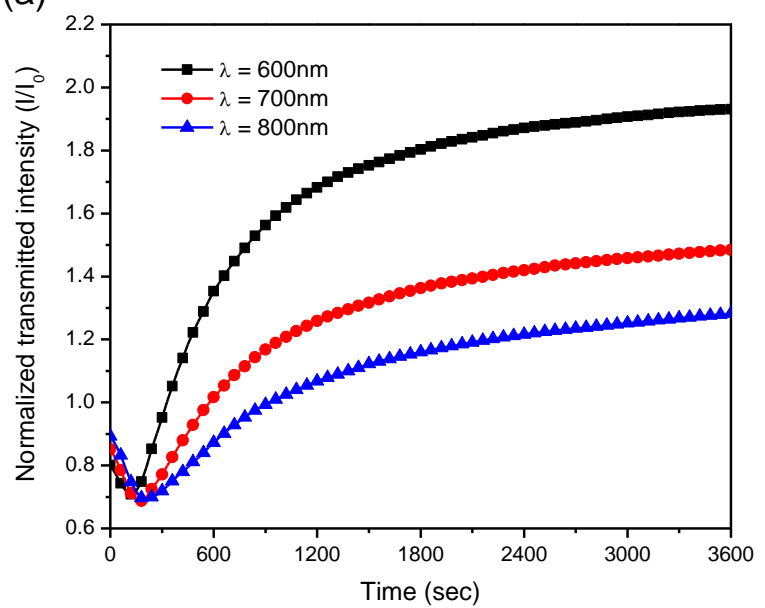

(b)

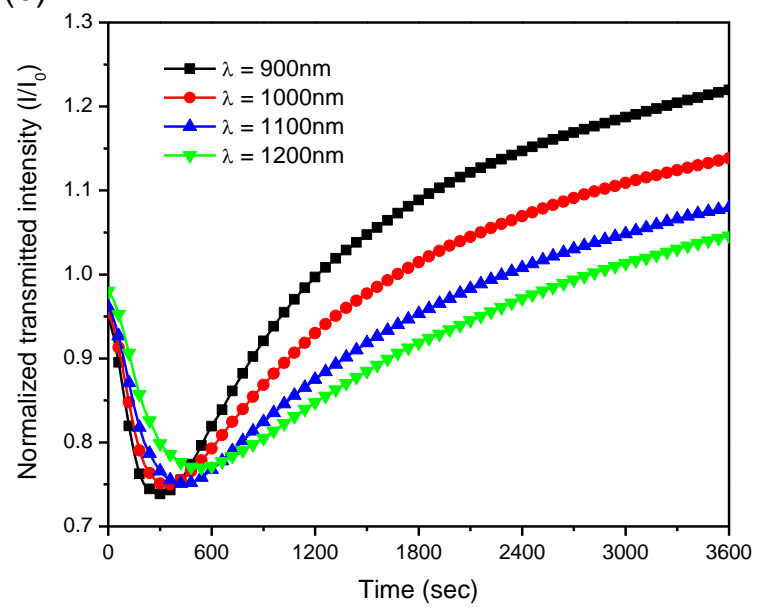

Fig. 3 Time evolution of transmitted intensity for $\varphi=0.005 \%$ ferrofluids at incident wavelength (a) $600 \mathrm{~nm}-800 \mathrm{~nm}$ and (b) $900 \mathrm{~nm}$ $1200 \mathrm{~nm}$ where the field intensity is 100 Gs [20].

Jin et al. [20] also investigated the wavelength dependence of magneto-optical transmission in ferrofluids. The suspensions contain $\mathrm{Fe}_{3} \mathrm{O}_{4}$ magnetic nanoparticles $(\sim 15 \mathrm{~nm})$ and the field intensity is 100 Gs. Similar to the results reported by Shulyma et al. [19, 37], ETI phenomena were also observed by them at different incident wavelengths as shown in Fig. 3. Interestingly, they found the dependence between characteristic time $\tau_{0}$ and incident wavelength can be described as $\tau_{0}$ $\sim \lambda^{\alpha}$, where the exponent $\alpha$ is equal to 2.07. Based on the FDTD simulation, the observed phenomena was explained quantitatively taking into consideration the aggregation process of the chainlike structures and the resonance inside the particle aggregates.

$\mathrm{Li}$ et al. [39] measured the transmittance of water-based $\mathrm{Fe}_{3} \mathrm{O}_{4}$ ferrofluids encapsulated in a sandwich-structured display (SSD) with and without alternating magnetic field (AMF). As showed in Fig. 4, the transmittance of $8.26 \mathrm{wt} \%$ ferrofluids increases remarkably from $1.3 \%$ to $83 \%$ in the presence of a 87.5 G AMF. Moreover, based on the changes in transmittance of the ferrofluids, it was also found that the concentration of the magnetic nanoparticles, frequency, and intensity of AMF had a great impact on the aggregation process of particle chains. 
(a)
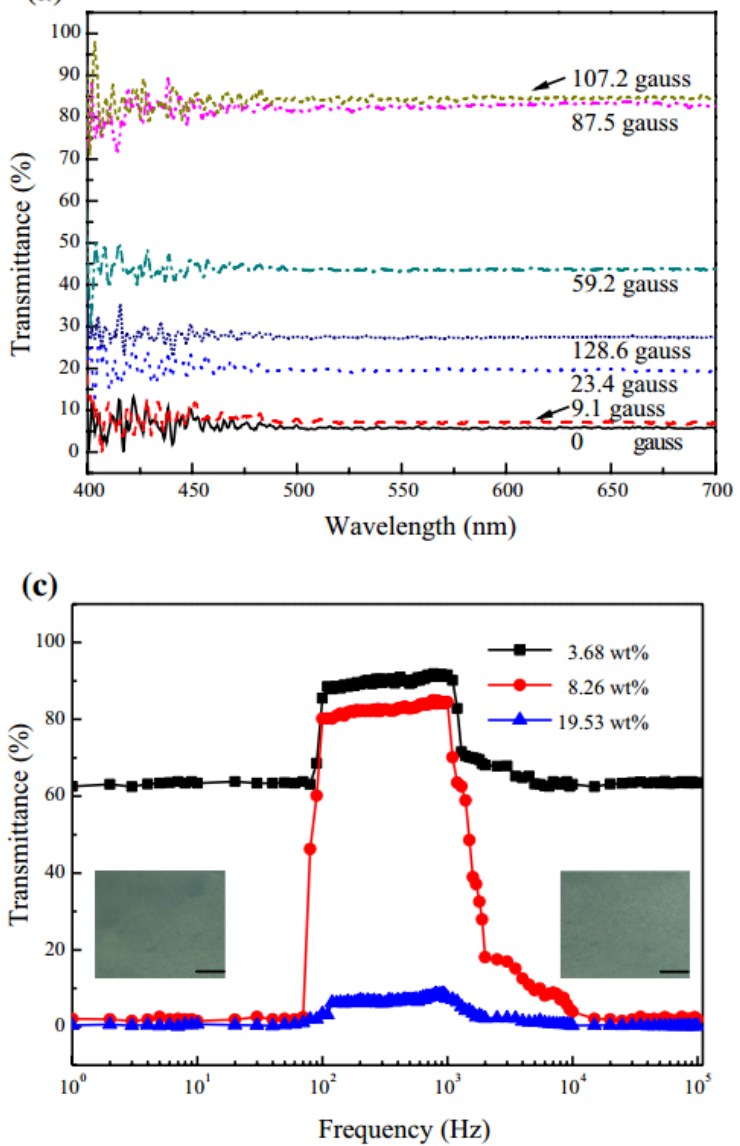

(b)

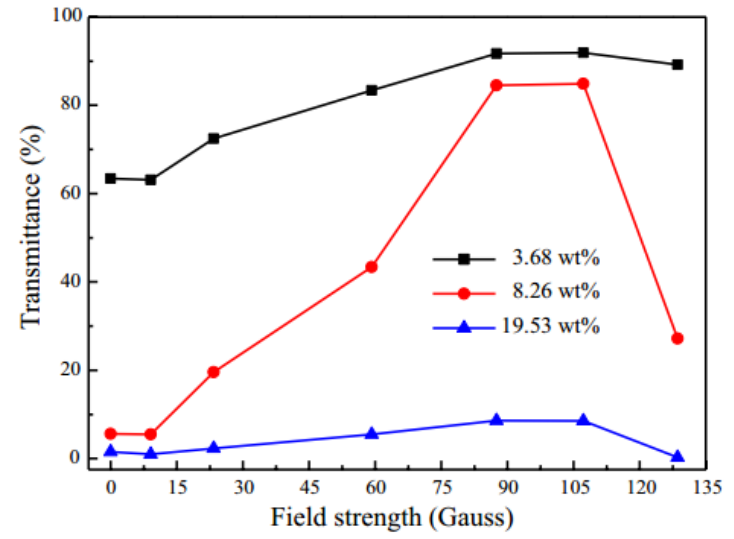

(d)

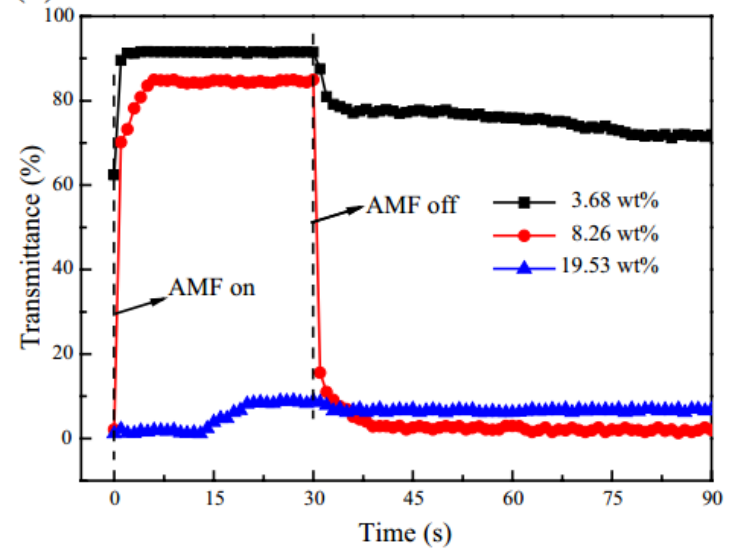

Fig. 4 (a) Transmittance of the ferrofluids vs. incident wavelength at different fiend intensity (a), vs. field strength at different particle concentrations (b), vs. field frequency at different particle concentrations (c), vs. time at different particle concentrations (d) [39].

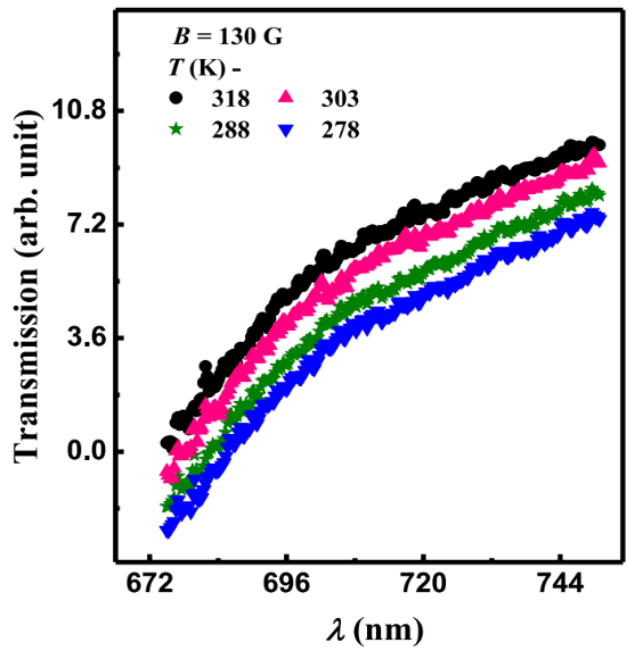

Fig. 5 Magneto-optical transmission vs. wavelength in ferrofluids at different temperatures where the field intensity is $130 \mathrm{G}$ [40].

Brojabasi et al. [40] systematically investigated the magneto-optical transmission in ferrofluids under different temperatures. As can be seen from Fig. 5, when the temperature is kept constant, the transmitted light intensity increases monotonically with the incident wavelength. It can be explained by the decreased extinction efficiency under larger incident wavelength based on the Rayleigh's scattering theory $\left(Q_{\text {sca }} \sim 1 / \lambda^{4}\right)$. Under the magnetic field, the parameter $L$ for two 
magnetized particles is described by Eq. (1). With the increasing of temperature, the parameter $L$ reduces and the rate of particle aggregation will become slower leading to the increased transmitted intensity as showed in Fig. 5. Besides, they found the light extinction rate reduced linearly with the increase of temperature, which should be attributed to the linearly increased diffusivity of the suspended particles.
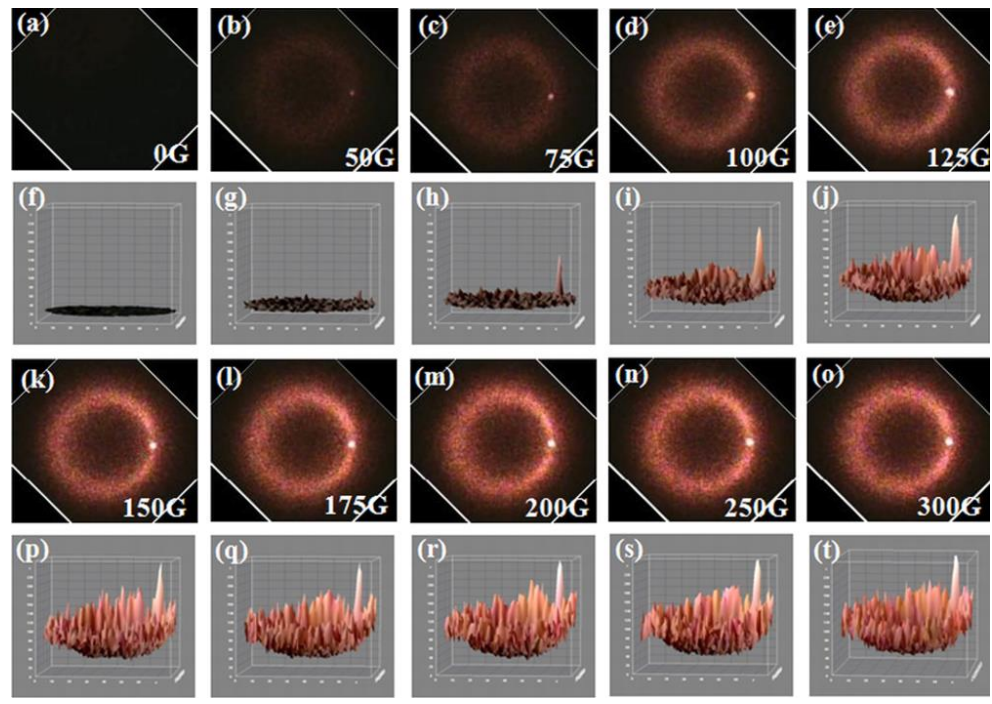

Fig. 6 Scattering patterns and corresponding 3-D surface plots from $\varphi=0.0042$ magnetic nanoemulsions at different field intensities [41].

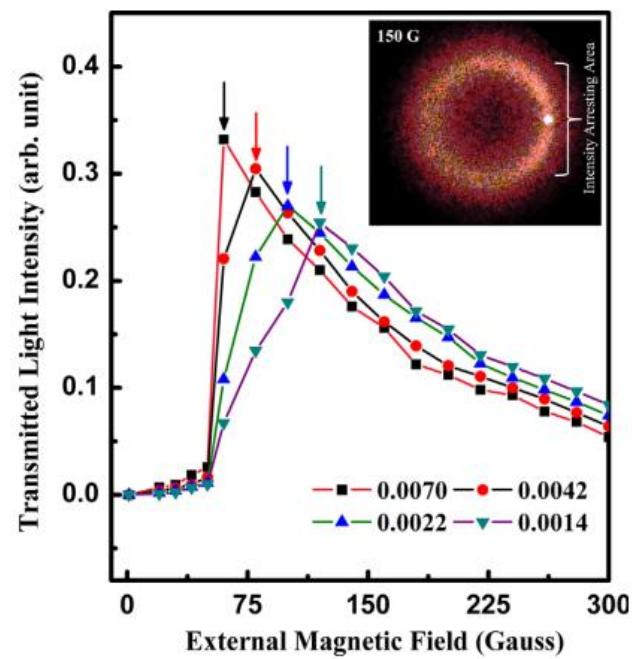

Fig. 7 Transmitted intensity vs. field intensity in magnetic nanoemulsions at different particle concentrations. Inset shows the ringlike structure for $\varphi=0.0042$ magnetic nanoemulsions at $B=150$ Gs [41].

Brojabasi et al. [41] also studied the field-dependent transmitted intensity and scattered pattern in magnetic nanoemulsions. Their main results are summarized in Figs. 6 and 7. When the field intensity is below 50 Gs, the sample keeps opaque resulted from its intense scattering as shown in Fig. 6. Beyond 50 Gs, the field induced particle aggregation will cause the increased size parameter where the extinction efficiency of Mie scattering reduces. As can be seen from Fig. 7, they reported that the transmitted intensity increased with the increasing of magnetic field until the field intensity reached a certain critical value and beyond which the intensity would inversely reduce. They explained this phenomenon should be 
due to the formation of waveguide-like aggregation structures. It was also observed by them that the critical field intensity would decrease at higher particle concentrations and such behavior could be described by a power law dependence, suggesting the occurrence of transition from disorder to order.

The absorption behavior at near infrared region in the same magnetic nanoemulsions was further studied by Brojabasi et al. [42]. It was found that the infrared absorption depended on both the sample concentration and the external field intensity. When the field intensity is less than $30 \mathrm{Gs}$, the absorption increase is not significant. For $B>30 \mathrm{Gs}$, a rapid increase was observed for absorption with the increase of field intensity. However, when the field intensity is larger than $80 \mathrm{Gs}$, the absorption will inversely decrease as showed in Fig. 8. Furthermore, the inset of Fig. 8 shows that the maximum absorption value increases with the increasing in photon energy while the corresponding field intensity decreases. These observed phenomena can be explained by the transition from Rayleigh scattering to Rayleigh-Mie transition.

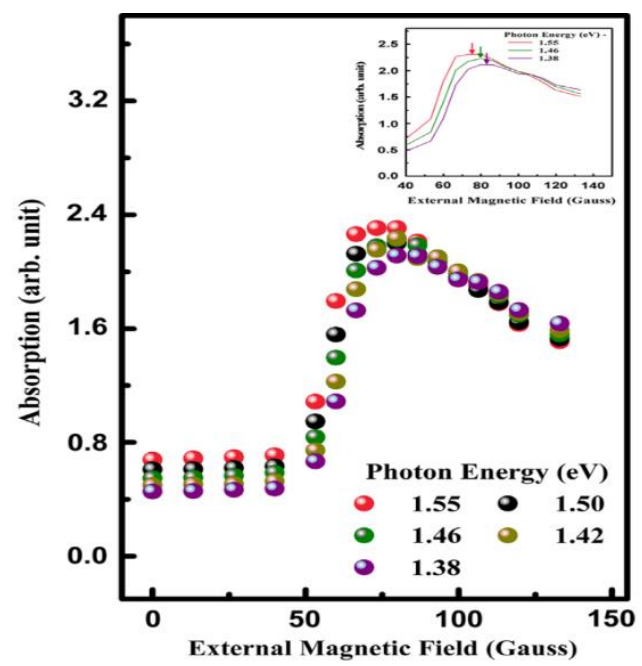

Fig. 8 Absorption vs. field intensity in $\varphi=0.0019$ magnetic nanoemulsion for different photon energies. Inset shows the continuous variations for $\mathrm{E}=1.55,1.46$, and $1.38 \mathrm{eV}[42]$.

As can be seen from the literatures introduced above, there are many studied conducted in recent years in regard to the light along the field direction. Various experimental parameters of these studies are summarized in Table 1 which indicates that the test conditions of them are different from each other including ferrofluid type, particle size, particle concentration, surfactant type, field type, field intensity, etc. Even so, we can obtain some coincident conclusions from their main outcomes listed in Table 2. For time-dependent investigations, it can be concluded that the transmitted intensity first decreases until the minimum and then it will reversely increase along with the time [17, 19, 20, 35-37]. This also indicates that the time evolution of transmitted intensity is of great importance for magneto-optical transmission phenomena in ferrofluids. Also, the dependence of transmitted light intensity on the field intensity was also studied extensively in the past several years. Results showed that beyond the critical field of certain value, the transmitted intensity firstly shows an increase trend while after the maximum it reduces with the field intensity [39-42]. However, it should be 
noted that the time effect was not considered for most field-dependent light transmission studies in the past. Therefore, it is strongly suggested that the combined effect of field intensity and time evolution investigate simultaneously in the future study.

Table 1 Summary of the experimental parameters for the light along the field direction in the literatures.

\begin{tabular}{|c|c|c|c|c|c|c|}
\hline Authors & Ferrofluid type & Size (nm) & Concentration (\%) & Surfactant & Field type & Field intensity (Gs) \\
\hline Huang et al. [35] & $\mathrm{CoFe}_{2} \mathrm{O}_{4} /$ water & 10 & $0.25,0.5$ & None & Gradient & $400-1300$ \\
\hline Li et al. [36] & $\left(\gamma-\mathrm{Fe}_{2} \mathrm{O}_{3} / \mathrm{Ni}_{2} \mathrm{O}_{3}\right) / \mathrm{HNO}_{3}$ & 10.59 & $3.76,9.17$ & None & Gradient & 300 \\
\hline \multirow{2}{*}{ Lin et al. [17] } & $\gamma-\mathrm{Fe}_{2} \mathrm{O}_{3} / \mathrm{Ni}_{2} \mathrm{O}_{3}(\mathrm{~A})$ & $11.21(\mathrm{~A})$ & $0.2(\mathrm{~A})$ & \multirow{2}{*}{ None } & \multirow{2}{*}{ Gradient } & \multirow{2}{*}{$500,900,1300$} \\
\hline & $\mathrm{Fe}_{2} \mathrm{O}_{3}(\mathrm{~B})$ & $6.54(\mathrm{~B})$ & $0,0.1,0.4,0.8(\mathrm{~B})$ & & & \\
\hline Shulyma et al. [19] & $\mathrm{Fe}_{3} \mathrm{O}_{4} /$ water & 11.5 & 1.2 & Oleic acid & Uniform & $0-3000$ \\
\hline Shulyma et al. [37] & $\mathrm{Fe}_{3} \mathrm{O}_{4} /$ kerosene & 11.5 & $0.2-2$ & Oleic acid & Uniform & $0-3000$ \\
\hline Jin et al. [20] & $\mathrm{Fe}_{3} \mathrm{O}_{4} /$ water & 15 & 0.005 & $\begin{array}{l}\text { Sodium oleate/ } \\
\text { PEG4000 }\end{array}$ & Uniform & 100 \\
\hline Li et al. [39] & $\mathrm{Fe}_{3} \mathrm{O}_{4} /$ water & 13.6 & $3.68,8.26,19.53$ & $\begin{array}{l}\text { 2- } \\
\text { mercaptoethanol }\end{array}$ & Alternating & $0-128.6$ \\
\hline Brojabasi et al. [40] & $\mathrm{Fe}_{3} \mathrm{O}_{4} /$ kerosene & 6.5 & 0.155 & Oleic acid & Uniform & $0-450$ \\
\hline Brojabasi et al. [41] & $\mathrm{Fe}_{3} \mathrm{O}_{4} /$ octane & 8 & $0.14,0.22,0.42,0.7$ & Oleic acid & Uniform & $0-350$ \\
\hline Brojabasi et al. [42] & $\mathrm{Fe}_{3} \mathrm{O}_{4} /$ octane & 8 & $0.14,0.19,0.22,0.67$ & Oleic acid & Uniform & $0-250$ \\
\hline
\end{tabular}

Table 2 Summary of the main outcomes for the light along the field direction in the literatures.

\begin{tabular}{|c|c|c|}
\hline Authors & Light scattering type & Main outcomes \\
\hline Huang et al. [35] & Time dependent & $\begin{array}{l}\text { When a low-gradient magnetic field was applied, it was found that the transmitted intensity first reduced to } \\
\text { the minimum and then it gradually increased to its initial value. Finally, the intensity either increased or } \\
\text { decreased slowly depended on the field gradient direction. }\end{array}$ \\
\hline Li et al. [36] & Time dependent & $\begin{array}{l}\text { Results show that the transmitted light through } \gamma-\mathrm{Fe}_{2} \mathrm{O}_{3} / \mathrm{Ni}_{2} \mathrm{O}_{3} \text { ferrofluid samples performs similar } \\
\text { relaxation behavior as other ferrofluids, indicating the formation of particle chains and their following } \\
\text { motion. }\end{array}$ \\
\hline Lin et al. [17] & Time dependent & $\begin{array}{l}\text { Their results indicate that the transmitted light tends to stabilize faster for binary ferrofluids with higher } \\
\text { volume fractions of weak magnetic particles. }\end{array}$ \\
\hline Shulyma et al. [19] & Time dependent & $\begin{array}{l}\text { The transmitted intensity first decreased rapidly and then recovered to its initial state even before the } \\
\text { disappearance the field pulse. The characteristic time for extinction inversion was found to be dependent on } \\
\text { both the magnetic pulse intensity and the samples thickness. }\end{array}$ \\
\hline Shulyma et al. [37] & $\begin{array}{l}\text { Both time and } \\
\text { wavelength dependent }\end{array}$ & The characteristic inversion time shifted to smaller values at lower incident wavelengths. \\
\hline Jin et al. [20] & $\begin{array}{l}\text { Both time and } \\
\text { wavelength dependent }\end{array}$ & $\begin{array}{l}\text { Interestingly, they found the dependence between characteristic time } \tau_{0} \text { and incident wavelength can be } \\
\text { described as } \tau_{0} \sim \lambda^{\alpha} \text {, where the exponent } \alpha \text { is equal to } 2.07 \text {. }\end{array}$ \\
\hline
\end{tabular}


The transmittance of $8.26 \mathrm{wt} \%$ ferrofluids increases remarkably from $1.3 \%$ to $83 \%$ in the presence of a 87.5

Li et al. [39] Field dependent

Brojabasi et al. [40] Temperature dependent

Brojabasi et al. [41] Field dependent

Brojabasi et al. [42] Field dependent
G AMF. Moreover, based on the changes in transmittance of the ferrofluids, it was also found that the concentration of the magnetic nanoparticles, frequency, and intensity of AMF had a great impact on the aggregation process of particle chains.

The light transmission of ferrofluids increased with the increasing in temperature.

When the field intensity is below 50 Gs, the sample keeps opaque resulted from its intense scattering. Beyond $50 \mathrm{Gs}$, they reported that the transmitted intensity increased with the increasing of magnetic field until the field intensity reached a certain critical value and beyond which the intensity would inversely reduce.

Under near infrared incident light, when the field intensity is less than $30 \mathrm{Gs}$, the absorption increase is not significant. For $B>30 \mathrm{Gs}$, a rapid increase was observed for absorption with the increase of field intensity. However, when the field intensity is larger than $80 \mathrm{Gs}$, the absorption will inversely decrease.

\subsection{Light normal to the field direction}

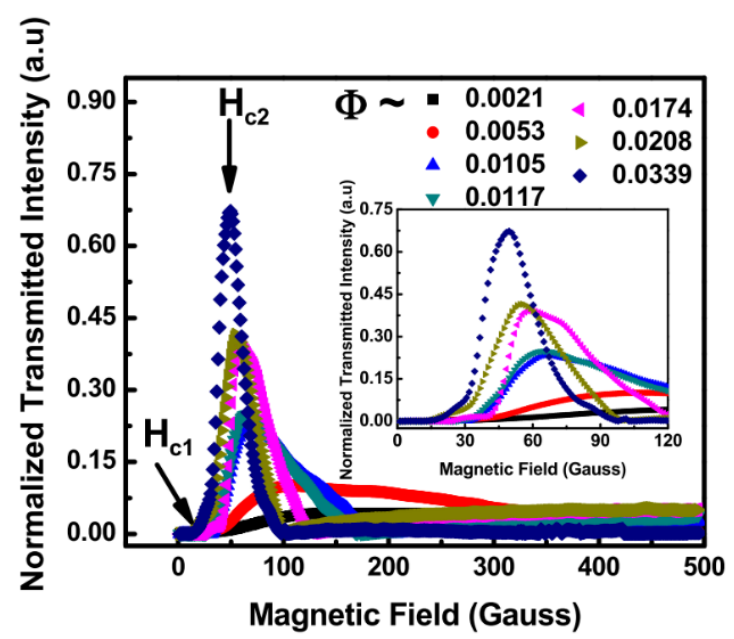

Fig. 9 Transmitted intensity vs. field intensity for magnetic nanoemulsions with different particle concentrations. Inset shows the blownup image at lower field intensities (0-120 Gauss) [43].

Most of the magneto-optical transmission studies related to the light normal to the field direction are presented in this section. Mohapatra and Philip [43] studied the magneto-optical transmission in magnetic nanoemulsions composed of $\sim 200$ nm droplets. As illustrated in Fig. 9, beyond a critical field intensity $\left(H_{\mathrm{c} 1}\right)$ and particle concentration $(\varphi>0.0021)$, they reported that the transmitted intensity was observed to increase rapidly until a maximum appears at the second critical field intensity $\left(H_{\mathrm{c} 2}\right)$. As the field intensity further increases, the transmitted intensity reversely reduces and finally it reaches a steady state value. What's more, they found that the maximum transmitted intensity at $H_{\mathrm{c} 2}$ increased linearly with volume fraction $\varphi$. Interestingly, both the two critical field intensities $H_{\mathrm{c} 1}$ and $H_{\mathrm{c} 2}$ was found to be dependent on volume fraction $\varphi$, namely, $H_{\mathrm{c}} \sim \varphi^{-x}$, with parameters 0.48 and 0.27 , respectively. They proposed that the optical birefringence should be the reason for the observed phenomena resulted from the rod-like aggregate structures. This conclusion can be verified by the speckle pattern recorded on the screen normal to the incident light as shown in Fig. 10. 


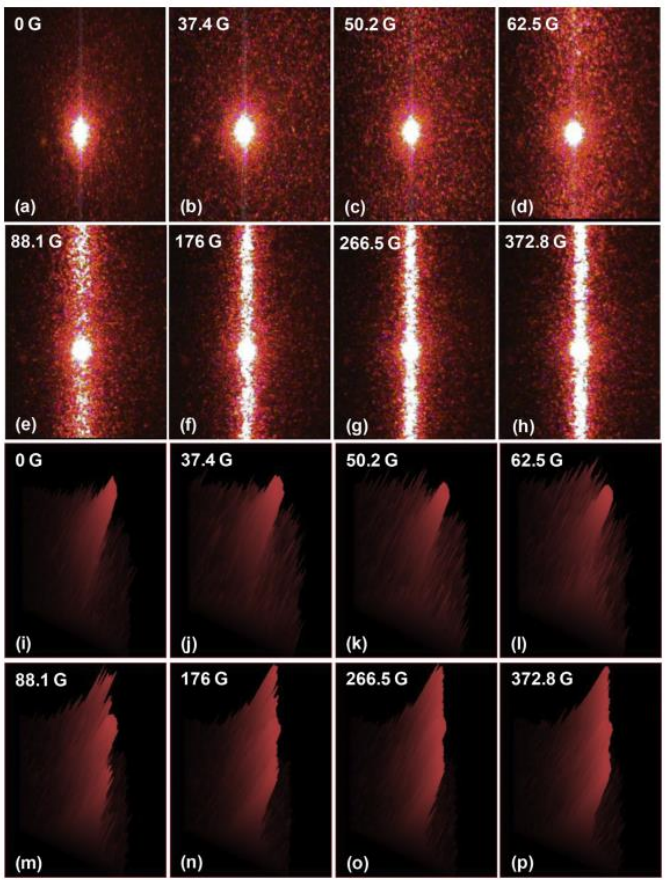

Fig. 10 Speckle patterns and their corresponding surface plots from $\varphi=0.0339$ magnetic nanoemulsions at different field intensities [43]

a
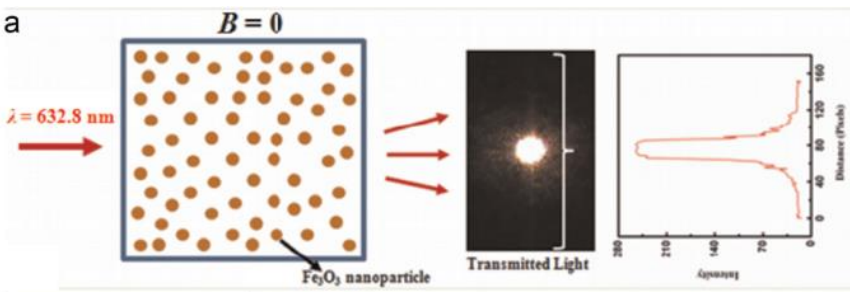

b

1 $B>B_{C 1}$
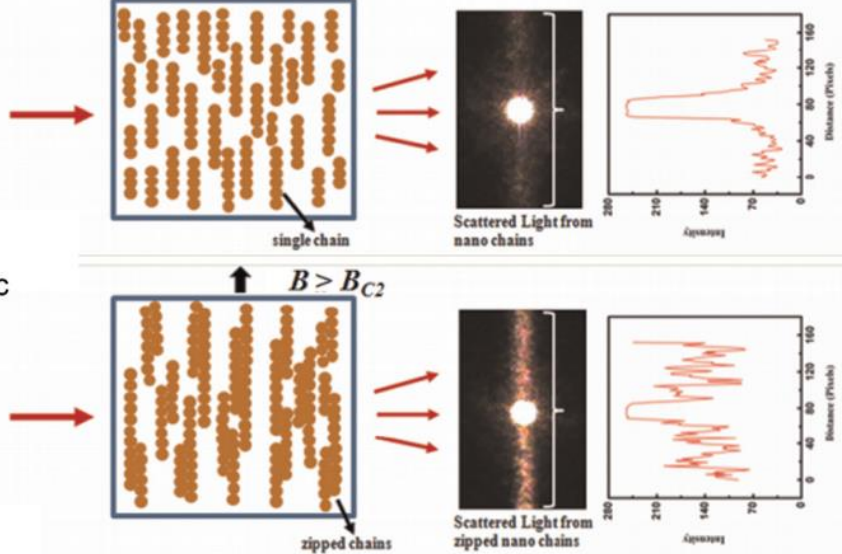

Fig. 11 The schematic diagram of the structural transition (left), the speckle pattern (middle) and the transmitted intensity distributions (right) for three different situations [44].

In another study, Brojabasi et al. [44] studied the magneto-optical transmission in ferrofluids with different $\mathrm{Fe}_{3} \mathrm{O}_{4}$ nanoparticle size. The diameters of the three nanoparticles are 15, 30 and $46 \mathrm{~nm}$, respectively. Without external field, there is only a bright circular spot on the screen. With the increasing in field intensity, a straight line scattering pattern appears containing spot and lobe parts. This can be explained by the aggregation process induced by external field as illustrated in Fig. 11. It was also found that the transmitted light intensity increased in the initial state until it reached the maximum at 
field intensity $B_{\mathrm{C} 1}$. Beyond $B_{\mathrm{C} 1}$, the transmitted intensity will inversely decrease with the field intensity and attained the minimum at the second field intensity $\left(B_{\mathrm{C} 2}\right)$. Moreover, as shown in Fig. 12, both critical field intensities were found to increase to higher values with the decrease of average size of the nanoparticles in ferrofluids. The authors proposed both the observed phenomena should be attributed to that at a constant particle concentration, ferrofluids with smaller particle size needs larger field intensity to form particle chains and the following column structures in the aggregation process.

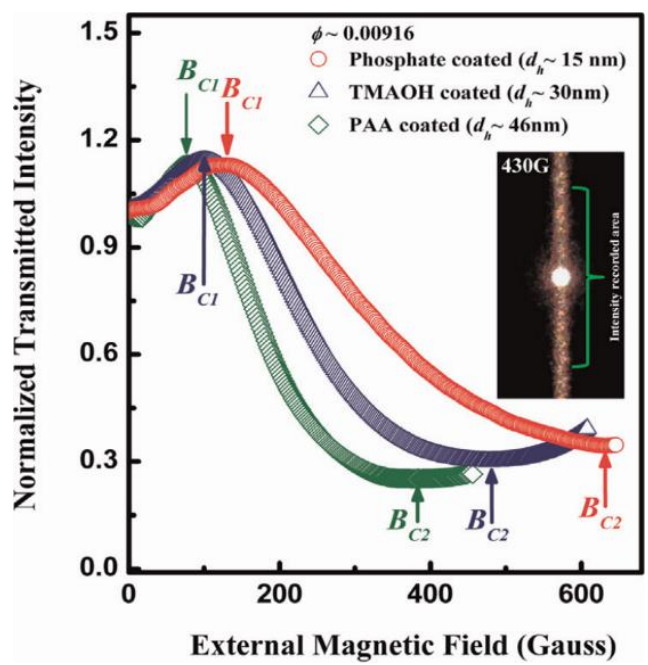

Fig. 12 Transmitted intensity vs. field intensity from ferrofluids with different particle sizes [44].

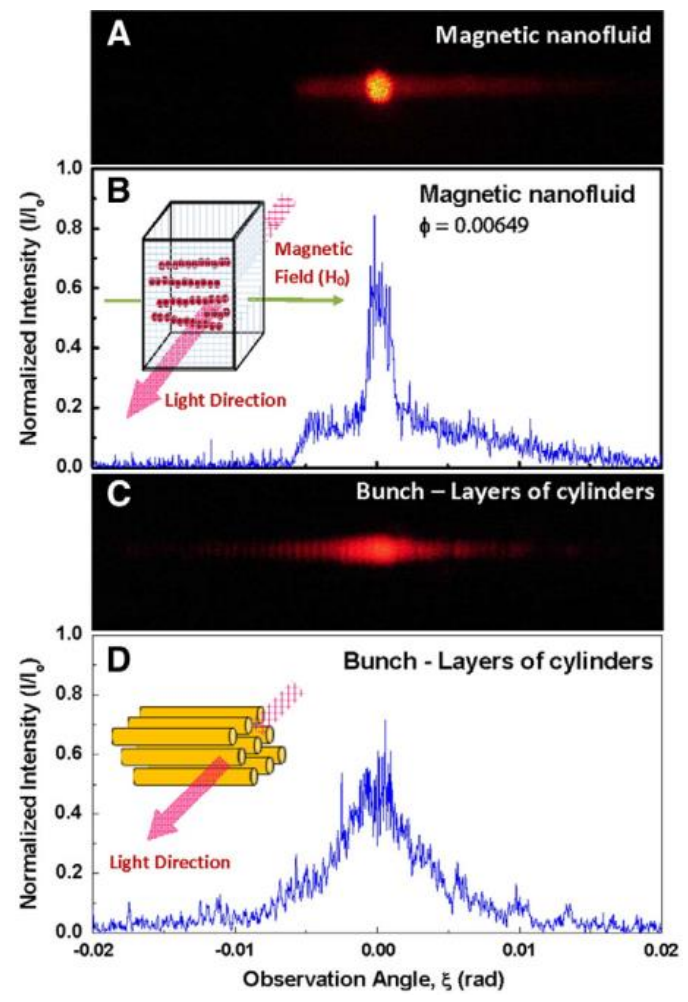

Fig. 13 Comparison of speckle patterns and intensity profiles vs. angle $(\xi)$ between magnetically polarized ferrofluids (A and B) and sixlayer cylinder clusters (C and D) [45].

Laskar et al. [45] compared the light scattering of field-induced magnetic nanoparticle chains with that of infinite cylinders. Figs. 13(a)-(d) show that the scattered patterns and intensity distributions in magnetically polarized ferrofluids 
and six-layer cylinder clusters respectively. As can be seen, there is a striking similarity of the scattered patterns between particle chains and macroscopic cylinders. They reported that, this is mainly attributed to the much smaller size of particle chains compared to the incident wavelength and thus the irregular aggregate structure behaves similarly as regular surfaces of infinite cylinders. However, the scattering pattern from macroscopic cylinder showed different-order diffraction fringes, whereas the scattering pattern from particle chains was diffused. They explained that the additional light diffraction effect in cylindrical surfaces should be responsible for the observed difference.

Some of the studies also considered the effect of direction of light polarization on the magneto-optical transmission phenomena in magnetic nanofluids. Desai et al. [46] investigated the influence of halloysite nanotubes (HNT) on the magneto-optical transmission in water-based ferrofluids consisting of $\mathrm{Fe}_{3} \mathrm{O}_{4}$ nanoparticles stabilized with lauric acid. The light's electric vector $(\mathbf{E})$ was set to be along the field direction $(\mathbf{H})$. At low HNT concentrations, namely, $0.5 \%$ and $2 \%$, they found that the magnetization of the suspensions increased remarkably, while further increasing HNT concentration to 2\% leaded to decreased magnetization. Results revealed that the existence of HNT facilitated the chain formation process, which was also confirmed by optical microscopy measurement and field-dependent diffraction effects.

Two light polarization directions were investigated simultaneously by Malynych and Moroz [47]. The results in Fig. 14 show that when the light polarization is normal to field direction $(\mathbf{E} \perp \mathbf{H})$, the relative transmitted intensity increases by certain degree due to the applied field. Then it reduces to a minimum value, beyond which it will increase slowly until reaching saturation. For $\mathbf{E} / / \mathbf{H}$, however, the transmitted intensity decreases by certain degree at first. A similar time evolution was observed in the following relaxation process. They proposed that the arrangement of magnetic moments along the field direction should be responsible for the different phenomena between $\mathbf{E} / / \mathbf{H}$ and $\mathbf{E} \perp \mathbf{H}$.

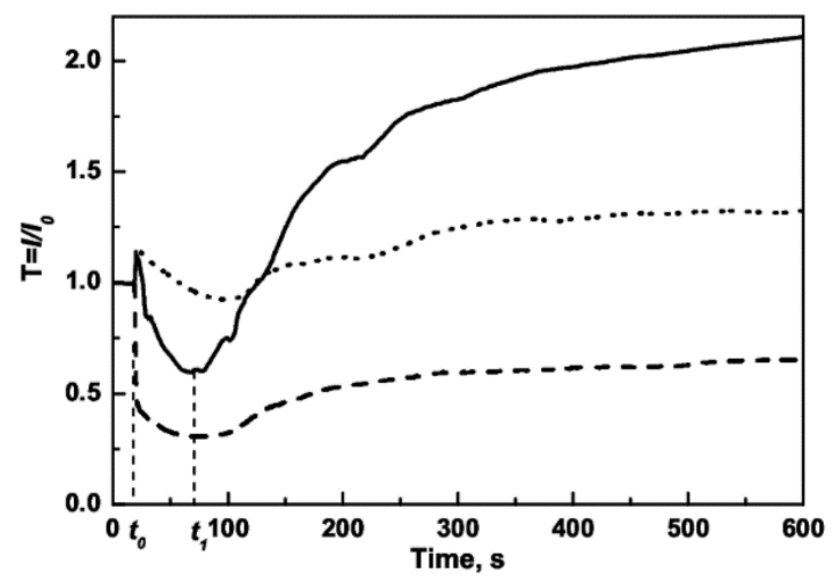

Fig. 14 Relative transmitted intensity vs. time for ferrofluids. Solid line represents light along the field direction; dotted - light normal to field direction, $\mathbf{E} \perp \mathbf{H}$; dashed - light normal to field direction, $\mathbf{E} / / \mathbf{H}$ [47].

Vales-Pinzón et al. [18] studied the time-dependent magneto-optical transmission in isoparaffin based ferrofluids in the presence of carbon nanotubes (CNTs). They investigated the light polarization along and normal to the field direction 
respectively. For $\mathbf{E} / / \mathbf{H}$, the transmitted intensity of all the suspensions was found to decrease in the first stage. Then, for suspensions without CNTs, a small increase and fluctuation in transmitted intensity were observed. On the contrary, for suspensions containing CNTs, the transmitted intensity increases significantly and finally it reaches a constant state value, which is depended on both the CNT volume fraction and the field intensity (Fig. 15). For $\mathbf{E} \perp \mathbf{H}$, it was observed that the addition of CNTs can significantly enhance the time variation of transmitted light intensity in ferrofluids. Moreover, they reported that the total increase of transmitted intensity was also enhanced due to the existence of CNTs. They concluded that the presence of CNTs should promote the chains formation and thus enhance the optical transmission.

a

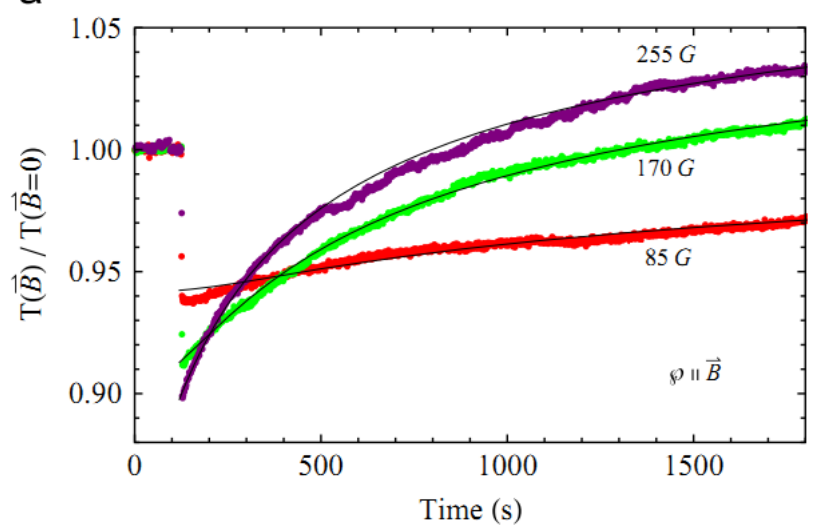

b

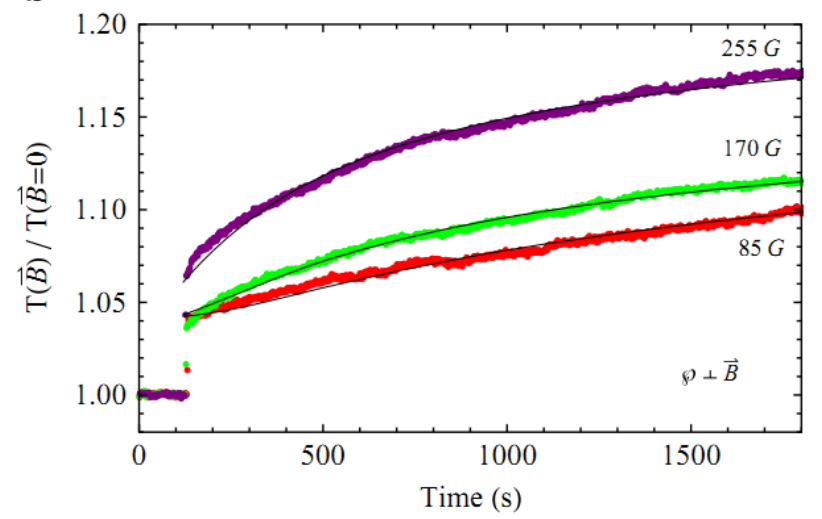

Fig. 15 Transmitted intensity vs. time of $0.1 \%$ ferrofluids composed of $0.05 \%$ carbon nanotubes for (a) $\mathbf{E} / / \mathbf{B}$ and (b) $\mathbf{E} \perp \mathbf{B}[18]$.

Socoliuc and Popescu [48] studied the magneto-optical transmission in ferrofluids under incident light with various polarization directions relative to that of magnetic field. The results indicate that the direction of light polarization can remarkably influence the magneto-optical tranmission in ferrofluids. When the angle of $\mathbf{E}$ vs. $\mathbf{H}$ is larger than $56^{\circ}$, the transmitted intensity increases compared to that without magnetic field while for other orientations, the transmitted intensity decreases for $\varphi=2 \%$. At high field intensity, the transmitted intensity shows a saturation tendency for all the cases. Moreover, they found that the optical extinction of light polarized at $54.74^{\circ}$ relative to the field direction was positive for the samples with low particle concentrations (2\%) and negative for the samples with high particle concentrations (4\% and $8 \%$ ), which was quite different from theoretically predicted result [49] that the particle orientation has negligible influence on the extinction of light polarized at $54.74^{\circ}$ relative to the field direction. They proposed that in lowconcentration samples, the chains formation under magnetic field is the dominant structuring mechanism resulted from interparticle interactions. In high-concentration ferrofluids, however, the dominant structuring mechanisms become the magnetic dipole-dipole interactions in the long range. As the result, the aggregated particle chains broken up to more isotropic structures such as coil-like or bundle-like chains.

A theoretical work was conducted by Fang et al. [50] to simulate the extinction coefficient of ferrofluids for light normal to the field direction by MD simulation. Fig. 16 shows the obtained results for light polarization along and normal 
to the field orientation, respectively. The extinction coefficients for the two cases were found to be quite different. As shown in Fig. 16(a), with magnetic field the extinction coefficient of ferrofluids was observed to be smaller compared to that without magnetic field for $\mathbf{E} \perp \mathbf{H}$. Results also indicate that the calculated extinction coefficient decreases with the field intensity. For $\mathbf{E} / / \mathbf{H}$ (Fig. 16(b)), however, they found the obtained extinction coefficient was larger compared to the no field condition. Their results agree well with the findings reported by Fosa et al. [51].
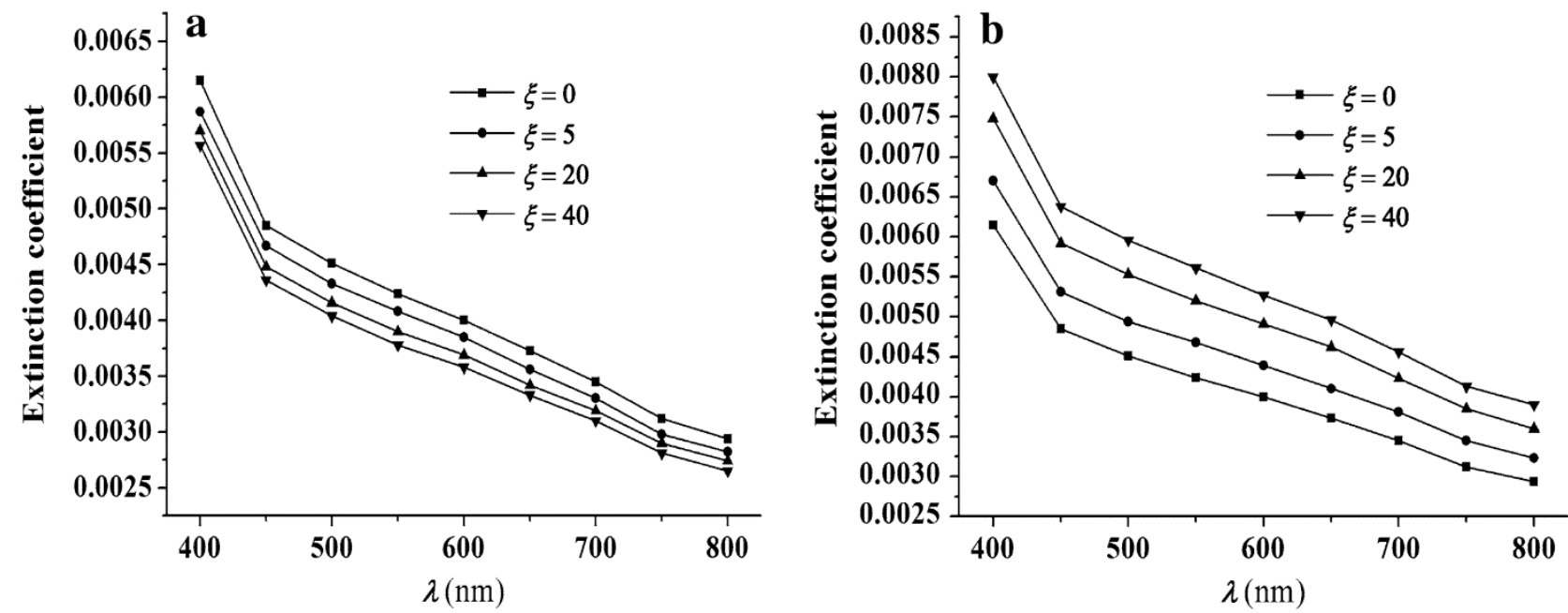

Fig. 16 Extinction coefficients of ferrofluids for (a) $\mathbf{E} \perp \mathbf{H}$ and (b) $\mathbf{E} / / \mathbf{H}$ where the light is normal to field direction [50].

Table 3 summarizes the experimental parameters for the incident light normal to the field direction from the recent literatures. It can be seen all of the studies employed the polarized light for light transmission measurements. Briefly their results listed in Table 4 show that the light polarization direction has a significant influence on the magneto-optical transmission in ferrofluids for the light normal to the field direction. General conclusions are the transmitted intensity will decrease when $\mathbf{E} / / \mathbf{H}$ while it will increase when $\mathbf{E} \perp \mathbf{H}$. However, there are disagreements among different studies with respect to time evolution of transmitted intensity in the presence of magnetic field. Therefore, further investigations for field-induced time-dependent light transmission in ferrofluids are still needed. Moreover, it should be mentioned that some of the studies $[43-45,52]$ did not declare the polarization direction though the polarized light was used as the light source. Due to the importance of polarization direction of light, we thus suggested that the polarization direction should take into consideration when polarized light was applied in the future study related to the magneto-optical transmission in ferrofluids for the light normal to the field direction.

Table 3 Summary of the experimental parameters for the light normal to the field direction in the literatures.

\begin{tabular}{lllllll}
\hline Authors & Ferrofluid type & Size $(\mathrm{nm})$ & Concentration $(\%)$ & Surfactant & Field type & Field intensity
\end{tabular}

\begin{tabular}{llllllll}
\hline Mohapatra and Philip [43] & $\mathrm{Fe}_{3} \mathrm{O}_{4} /$ octane & 10 & $0.21-3.39$ & Oleic acid & Uniform & $0-500$ & Polarized light \\
\hline
\end{tabular}




\begin{tabular}{|c|c|c|c|c|c|c|c|}
\hline Brojabasi et al. [44] & $\mathrm{Fe}_{3} \mathrm{O}_{4} /$ water & $15,30,46$ & 0.916 & $\begin{array}{l}\text { PAA, TMAOH, } \\
\text { phosphate }\end{array}$ & Uniform & $0-600$ & Polarized light \\
\hline Laskar et al. [45] & $\mathrm{Fe}_{3} \mathrm{O}_{4} /$ hydrocarbon & 6.5 & 0.649 & Oleic acid & Uniform & 3768 & Polarized light \\
\hline Desai et al. [46] & $\mathrm{Fe}_{3} \mathrm{O}_{4} /$ water & 8.4 & 1.4 & Lauric acid & Uniform & $0-1800$ & $\mathbf{E} / / \mathbf{H}$ \\
\hline Malynych and Moroz [47] & $\mathrm{Fe}_{3} \mathrm{O}_{4} /$ water & 10 & 0.12 & None & Uniform & 7200 & $\mathbf{E} / / \mathbf{H}, \mathbf{E} \perp \mathbf{H}$ \\
\hline Vales-Pinzón et al. [18] & $\mathrm{Fe}_{3} \mathrm{O}_{4} /$ isoparaffin & 10 & 0.1 & Not clear & Uniform & $85,170,255$ & $\mathbf{E} / / \mathbf{H}, \mathbf{E} \perp \mathbf{H}$ \\
\hline Socoliuc and Popescu [48] & $\mathrm{Fe}_{3} \mathrm{O}_{4} /$ oil & 6.3 & $2,4,8$ & Oleic acid & Uniform & $0-3500$ & Various $\mathbf{E}$ vs. $\mathbf{H}$ \\
\hline
\end{tabular}

Table 4 Summary of the main outcomes for the light normal to the field direction in the literatures.

Authors Light scattering type Main outcomes

Beyond a critical field intensity $\left(H_{\mathrm{c} 1}\right)$ and particle concentration $(\varphi>0.0021)$, they reported that the transmitted intensity was observed to increase rapidly until a maximum appears at the second critical

Mohapatra and Philip [43] Field dependent

Brojabasi et al. [44]

Laskar et al. [45]

Desai et al. [46]

Malynych and Moroz [47] Time dependent

Vales-Pinzón et al. [18] Time dependent

Socoliuc and Popescu [48] Field dependent

Fang et al. [50]

Field dependent

Field dependent field intensity $\left(H_{\mathrm{c} 2}\right)$. As the field intensity further increases, the transmitted intensity reversely reduces and finally it reaches a steady state value. Interestingly, both the two critical field intensities $H_{\mathrm{c} 1}$ and $H_{\mathrm{c} 2}$ was found to be dependent on volume fraction $\varphi$, namely, $H_{\mathrm{c}} \sim \varphi^{-x}$.

It was found that the transmitted light intensity increased in the initial state until it reached the maximum at field intensity $B_{\mathrm{Cl}}$. Beyond $B_{\mathrm{Cl}}$, the transmitted intensity will inversely decrease with the field intensity and attained the minimum at the second field intensity $\left(B_{\mathrm{C} 2}\right)$. Both critical field intensities were found to increase to higher values with the decrease of average size of the nanoparticles in ferrofluids.

There is a striking similarity of the scattered patterns between particle chains and macroscopic cylinders.

The addition of nanotubes of halloysite to ferrofluids can facilitate the field-induced chain formation process.

For $\mathbf{E} \perp \mathbf{H}$, the relative transmitted intensity increases by certain degree due to the applied field. For $\mathbf{E}$ $/ / \mathbf{H}$, however, the transmitted intensity decreases by certain degree at first.

For $\mathbf{E} / / \mathbf{H}$, the transmitted intensity of all the suspensions was found to decrease in the first stage. Then, for suspensions without CNTs, a small increase and fluctuation in transmitted intensity were observed. On the contrary, for suspensions containing CNTs, the transmitted intensity increases significantly and finally it reaches a constant state value, which is depended on both the CNT volume fraction and the field intensity. For $\mathbf{E} \perp \mathbf{H}$, it was observed that the addition of CNTs can significantly enhance the time variation of transmitted light intensity in ferrofluids.

The results indicate that the direction of light polarization can remarkably influence the magneto-optical tranmission in ferrofluids. Moreover, they found that the optical extinction of light polarized at $54.74^{\circ}$ relative to the field direction was positive for the samples with low particle concentrations $(2 \%)$ and negative for the samples with high particle concentrations ( $4 \%$ and $8 \%$ ).

With magnetic field the extinction coefficient of ferrofluids was observed to be smaller compared to that without magnetic field for $\mathbf{E} \perp \mathbf{H}$. For $\mathbf{E} / / \mathbf{H}$, however, they found the obtained extinction coefficient was larger compared to the no field condition.
Field and wavelength dependent 


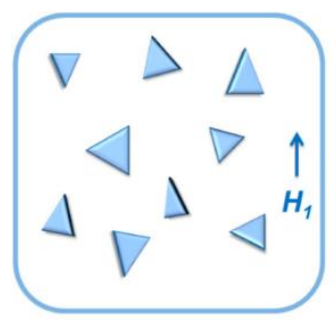

Low LT

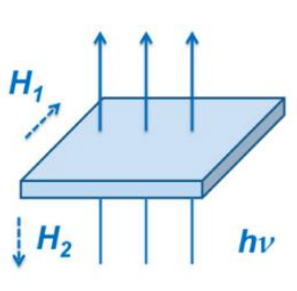

hv

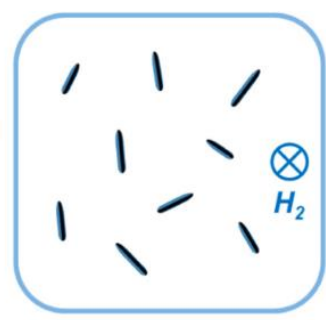

High LT

Fig. 17 Schematic diagram of controlling the transmittance of ferrofluids by response of Ag nanoplates [53].

In this section, the magneto-optical transmission in ferrofluids for other cases is reviewed. The transmittance of ferrofluids composed of $\mathrm{Fe}_{3} \mathrm{O}_{4}$ nanoparticles and $\mathrm{Ag}$ nanoplates was studied by Mao et al. [53]. They reported that the variation of light transmittance (LT) of the suspensions can be controlled by the movement of Ag nanoplates. For external field normal to the light direction, the triangular faces of Ag nanoplates will turn toward the incident light. As a result, the incident light is blocked leading to the decreased transmittance (low LT mode). For external field along the light direction, however, the edges of Ag nanoplates will turn toward the incident light. In such condition, the reflection, scattering, and absorption of Ag nanoplates reach minimum resulting in the increased transmittance (high LT mode). The schematic diagram for the two modes is shown in Fig. 17. Besides, the transmittance variation between the two modes was found to be dependent on the field intensity (Fig. 18). It can be seen the value of $\Delta(\mathrm{LT})$ increases with the field intensity and tends to get saturation finally. Moreover, the switching of transmittance of ferrofluids by external field was demonstrated to be rapid and perfectly reversible.
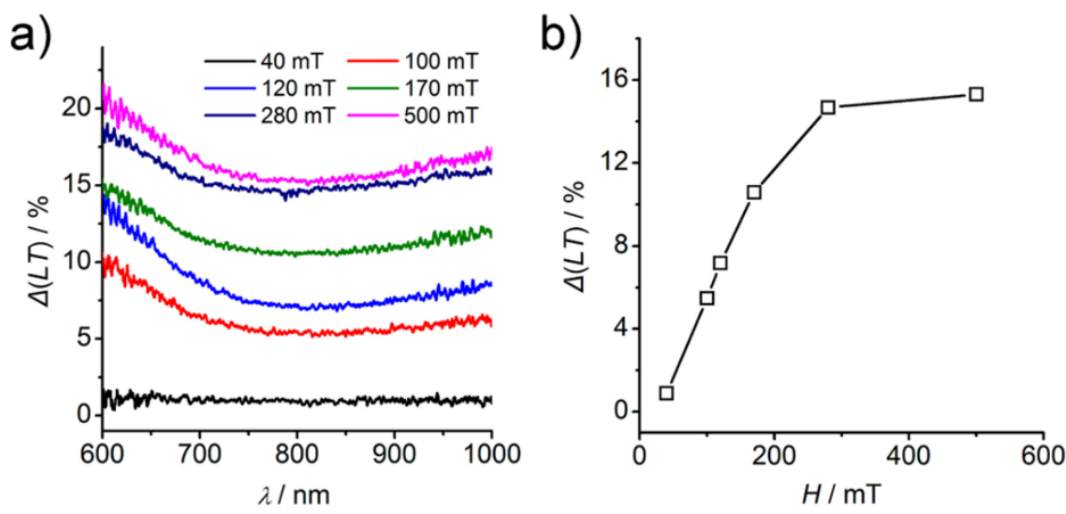

Fig. 18 (a) Transmittance variation $\Delta(\mathrm{LT})$ vs. wavelength for ferrofluids containing Ag nanoplates at different field intensities. (b) The dependence of field intensity on $\Delta(\mathrm{LT})$ where the wavelength is $800 \mathrm{~nm}$ [53].

Lv et al. [54] employed the Monte Carlo method to simulate the field induced particle aggregation structures and calculate the transmittance of ferrofluids at different situations. After comparison with the experimental data, the numerical method adopted in their study was demonstrated to be valid. Then the transmittance of ferrofluids was calculated and discussed by the authors in detail. From Fig. 19, the results shows that at $1550 \mathrm{~nm}$ wavelength, the transmittance for light along and normal to the field direction was the maximum and minimum respectively while the transmittance for no field 
situation was in the middle.

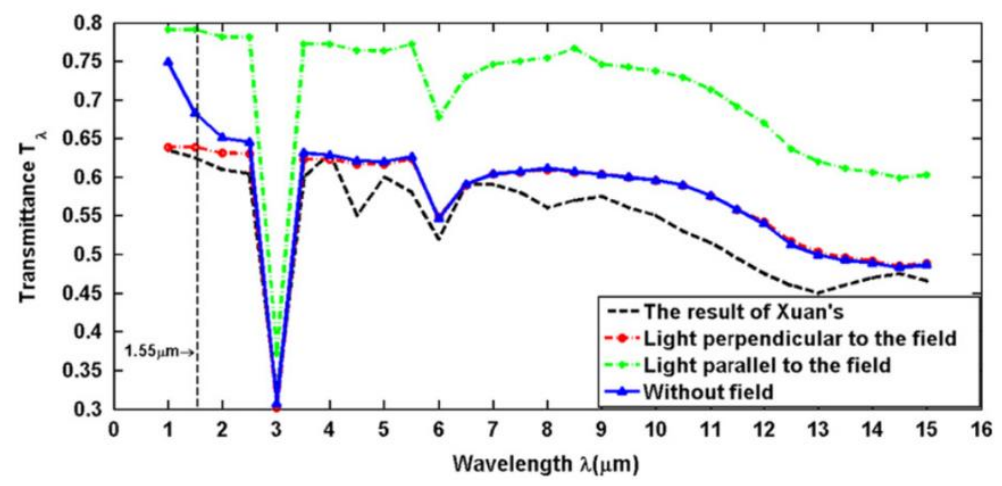

Fig. 19 The calculated transmission spectrum of ferrofluids [54].

Kushnir et al. [55] synthesized the hard-magnetic plate-like $\mathrm{SrFe}_{12} \mathrm{O}_{19}$ nanoparticles and studied the magneto-optical transmission of the prepared suspensions under various configurations of incident light $\mathbf{K}$, magnetic field $\mathbf{H}$ and electric field vector of light E. The results are illustrated in Fig. 20. Fig. 20(a) shows the optical transmittance of ferrofluid suspensions under different angles $\alpha$ between $\mathbf{E}$ and $\mathbf{H}$ for the case $\mathbf{H} \perp \mathbf{K}$. As can be seen, the transmittance decreases continuously by an order of magnitude as the angle $\alpha$ increases from $0^{\circ}$ (E // H) to $90^{\circ}$ ( $\left.\mathbf{E} \perp \mathbf{H}\right)$. Fig. 20(b) plots the transmittance vs. field strength for both polarized and unpolarized light. It indicates that the optical transmittance depends on the mutual directions among $\mathbf{K}, \mathbf{E}$ and $\mathbf{H}$. They concluded that this phenomenon is due to the different nanoparticle alignments.
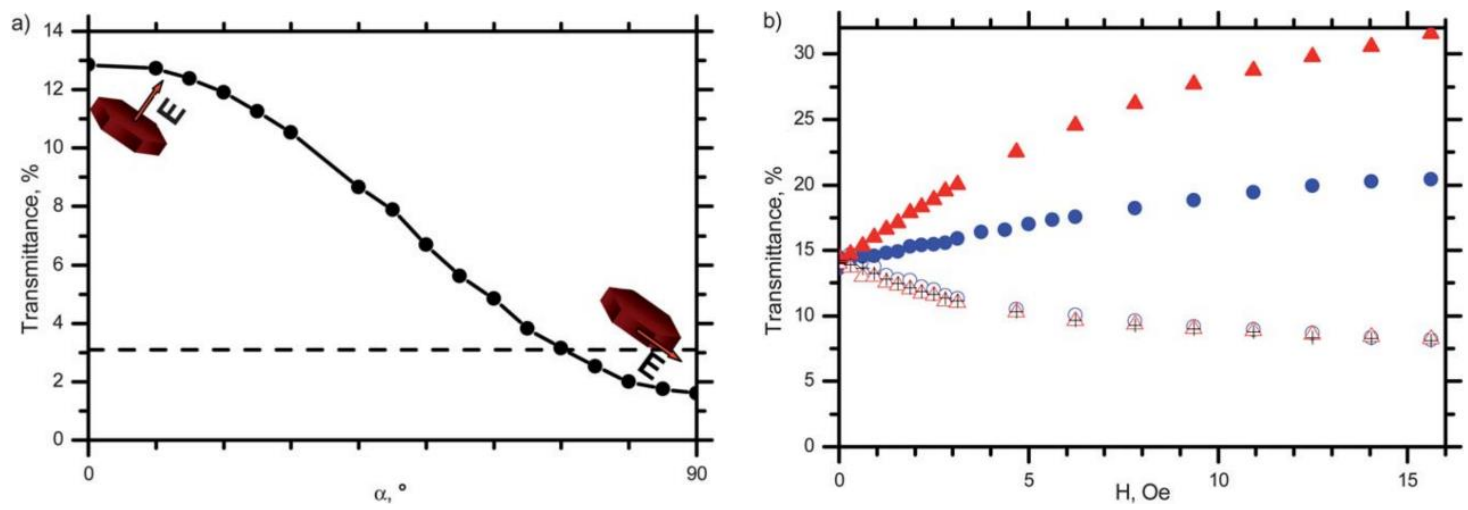

Fig. 20 (a) Transmittance of ferrofluids containing $\mathrm{SrFe}_{12} \mathrm{O}_{19}$ nanoparticles for various orientations between field $\mathbf{H}$ and light $\mathbf{E}$ vector. Solid line represents 14 Oe field; dashed line represents no field. (b) Transmission vs. field intensity for $550 \mathrm{~nm}$ wavelength and 114 Oe field intensity. Open and solid circles represent $\mathbf{H} / / \mathbf{K}$ and $\mathbf{H} \perp \mathbf{K}$ for unpolarized light respectively; Cross, open and solid triangles represent (i) $\mathbf{H} / / \mathbf{K}$, (ii) $\mathbf{H} \perp \mathbf{E}$ and $\mathbf{H} \perp \mathbf{K}$, and (iii) $\mathbf{H} / / \mathbf{E}$ and $\mathbf{H} \perp \mathbf{K}$ for polarized light respectively [55].

\section{Applications}

Due to the magnetic field tunability of optical properties, the ferrofluids can find numerous applications in different fields, such as tunable optical filter [56], optical grating [57], optical limiter [58], optical fiber modulator [59, 60], optical switch [61], magneto optical wavelength filter [62, 63], optical fiber sensor [64], etc. Here, we discuss some of the most recent studies related to the magneto-optical applications of ferrofluids, since the old ones have already been covered in 
the earlier reviews.

Li et al. [39] proposed a novel field-induced displays based on the change of transmittance of ferrofluids under the alternating magnetic field (AMF). After encapsulating the ferrofluids in a sandwich-like structure as shown in Fig. 21, they measured the transmittance of light through ferrofluids for the light along the field direction (Fig. 4). Furthermore, the performance of the proposed device was tested by an iron sheet hollowed by letters "NTUST". As can be seen from Fig. 22(a), in the absence of external field, the photograph remains complete opaque, suggesting that the incident light cannot transmit through the ferrofluids completely due to the well-dispersed magnetic nanoparticles. After the magnetic field was turned on, it was found the nanoparticles would aggregate into chainlike structures, leading to the increased transmittance. Therefore, in Fig. 22(b) the letters "NTUST" can be clearly recognized on account of the contrast difference between black and transparent regions.

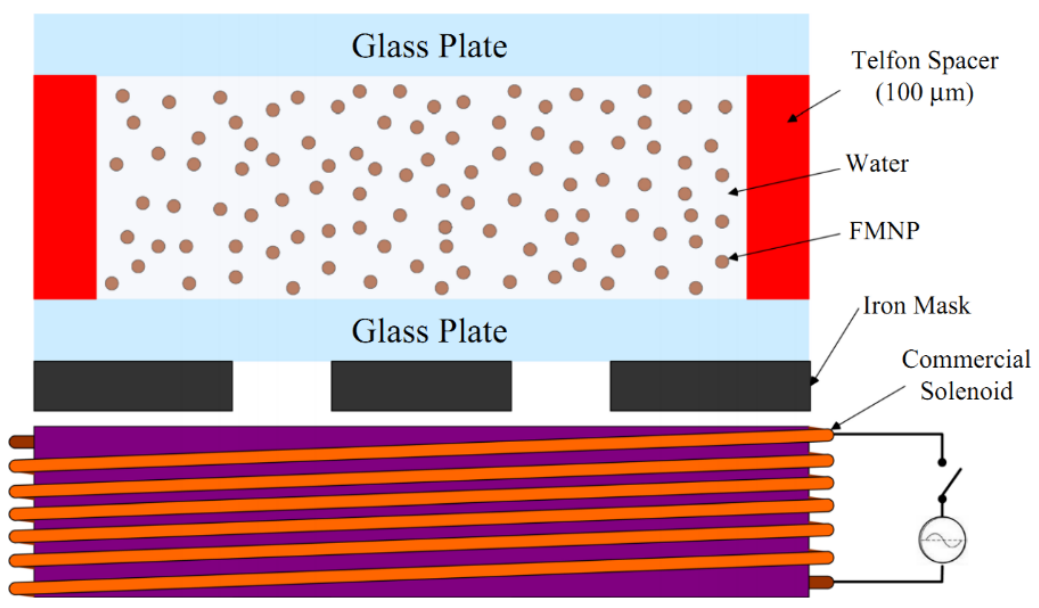

Fig. 21 Schematic diagram of the novel field-induced displays based on the magneto-optical transmission in ferrofluids [39]
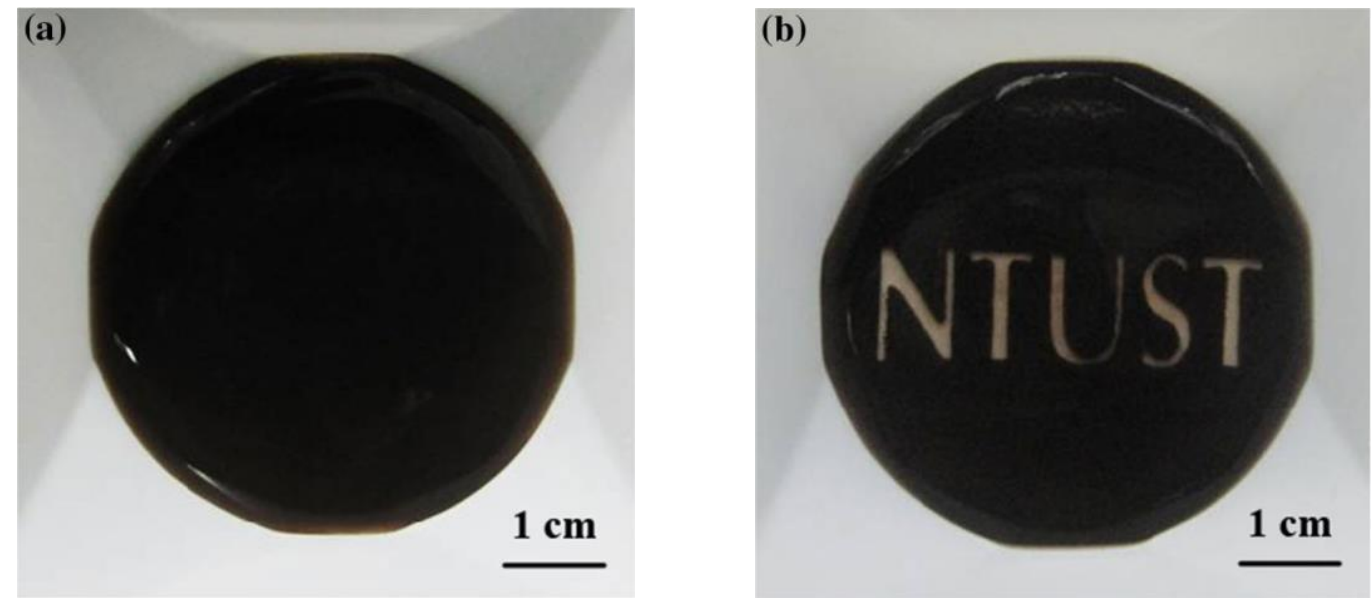

Fig. 22 Images of the novel field-induced displays (a) without and (b) with magnetic field [39].

Gimenez et al. [65] proposed a simple, rapid and low-cost method to determine the concentration of ferrofluids with low particle contents by the variation of light transmittance and scattering in ferrofluids. The schematic diagram of this setup is shown in Fig. 23 where the light is normal to the field direction. For both 0 D nanoparticles and 1 D nanowires, the intensity of scattering signal was found to be stronger than that of transmission signal. Take small nanowires for example, 
as can be seen from Fig. 24(a), the scattered signal can be captured by detectors even at very low particle amounts such as $2 \mathrm{ng} \mathrm{mL} \mathrm{mL}^{-1}$. However, the transmitted signal cannot be detected at such a low concentration (Fig. 24(b)). Besides the high sensitivity to determine the content of nanoparticles, their proposed method is also very promising to detect the geometry of the magnetic nanomaterial in the suspensions.

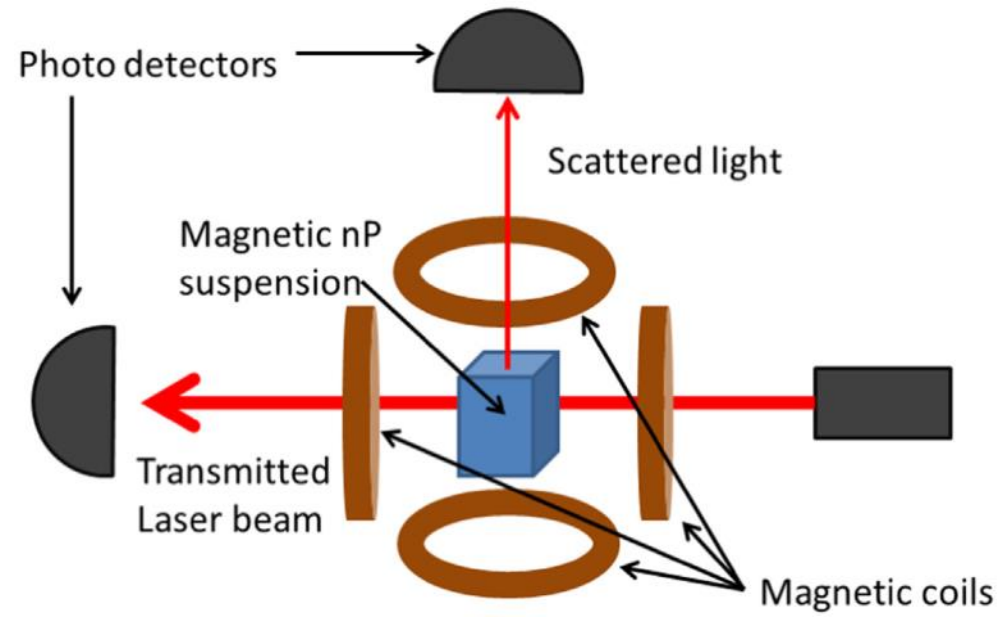

Fig. 23 Schematic diagram of the setup used to determine the concentration of ferrofluids with low particle contents [65].

a

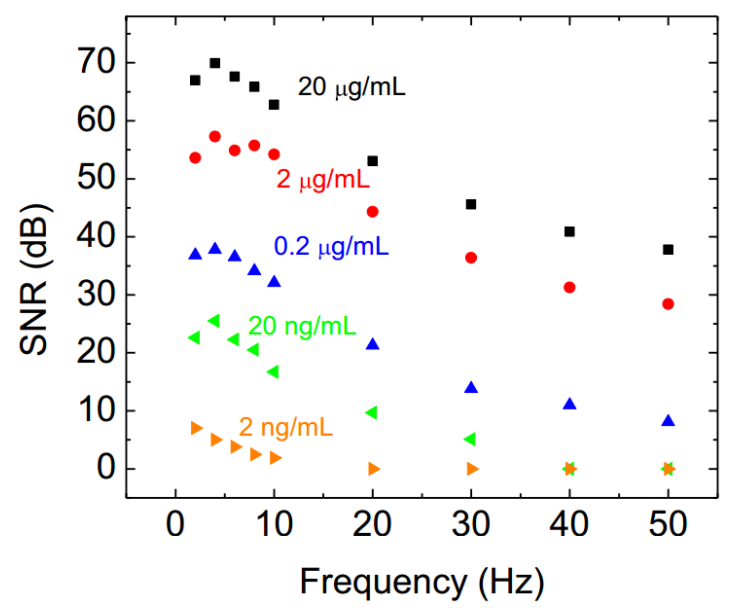

b

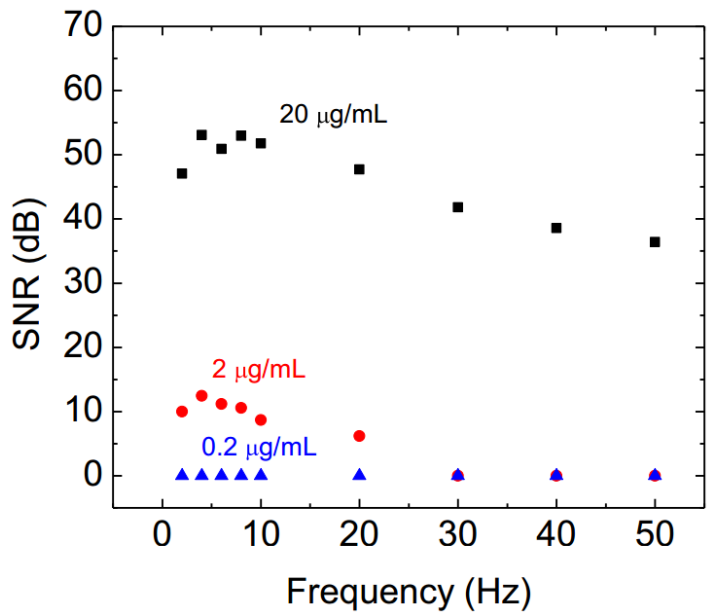

Fig. 24 Signal-to-noise ratio (SNR) vs. field frequency for ferrofluids with different particle contents determined by (a) scattered signal and (b) transmitted signal [65].

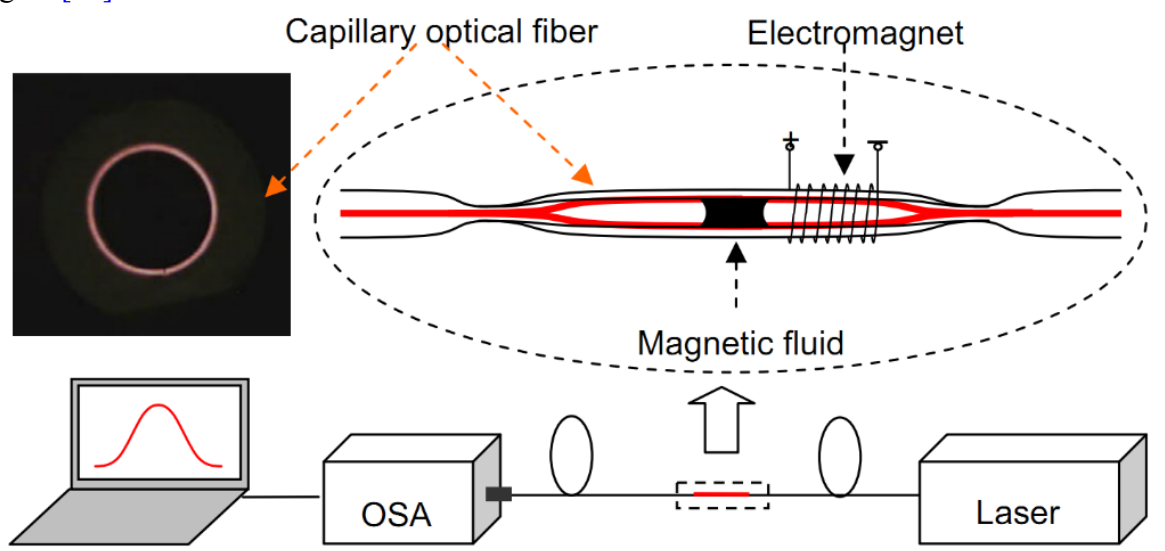

Fig. 25 Schematic diagram of the optical capillary modulator based on magneto-optical transmission in ferrofluids [66]. 
A novel ferrofluid-based optical capillary modulator was developed by Yang et al. [66]. The ferrofluids were placed into a capillary optical fiber as shown in Fig. 25. Results demonstrated that an incident beam at 632.8 nm wavelength can be modulated by only $2.17 \times 10^{-2} \mu \mathrm{L}$ ferrofluids under magnetic field. Fig. 26 shows the time evolution of the modulation property of the proposed device under different field intensity. Through optimizing the particle concentration, temperature and field intensity, they obtained a wide modulation-depth ranging from $44 \%$ to $75 \%$. Besides, the proposed modulator exhibited good stability and repeatability for practical applications.

a

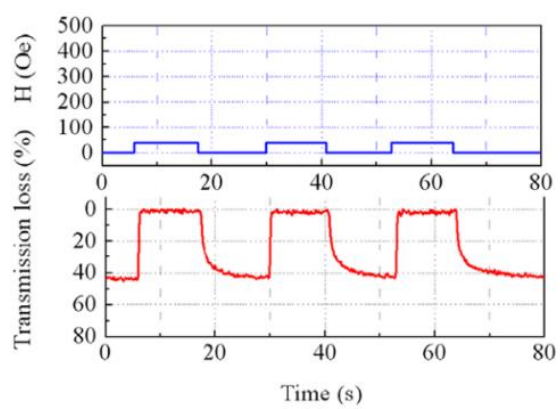

b

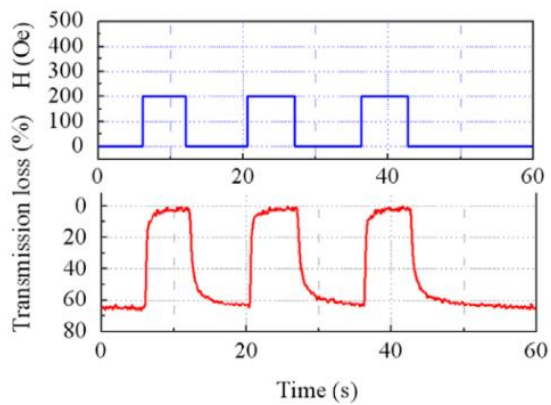

C

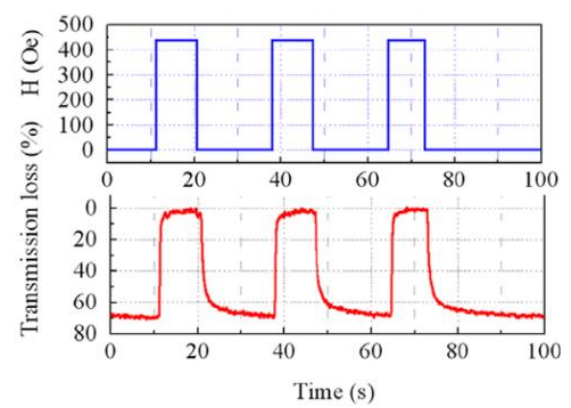

Fig. 26 Transmission loss vs. time for the optical modulator based on ferrofluids at field intensity (a) 40 Oe; (b) 200 Oe; (c) 440 Oe [66]. Magnetic field

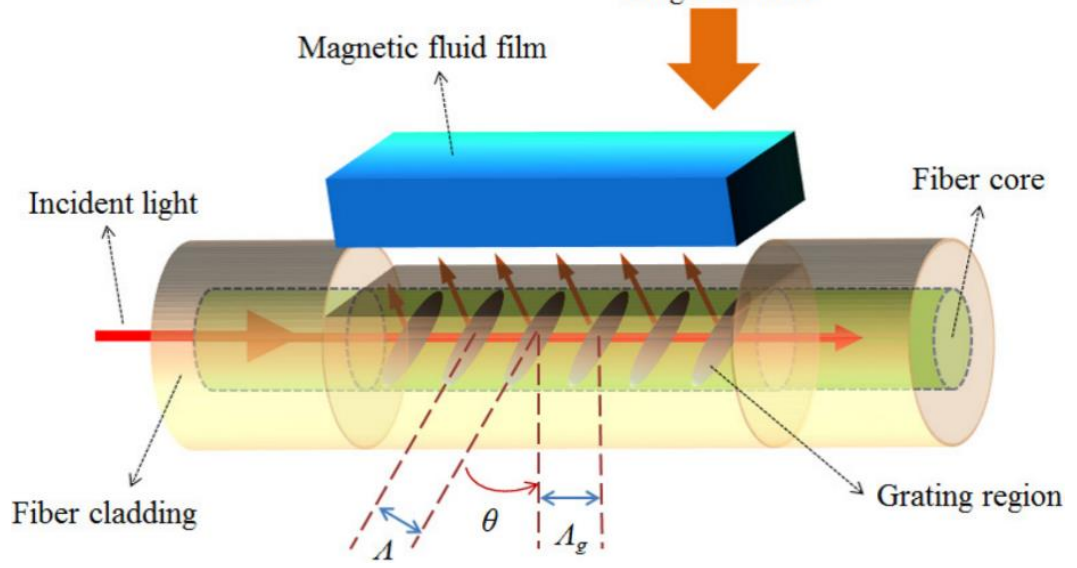

Fig. 27 Schematic diagram of ferrofluid-based field intensity sensor using D-shaped Bragg grating [67].

The ferrofluid-based magnetic field fiber sensors were widely studied recently [67-70]. Lin et al. [68] reported a theoretical model of a field intensity sensor composed of ferrofluids combined with tilted fiber Bragg grating. In their study, they investigated the influence of tilt angle and field intensity on the transmission properties of the proposed sensor. It was found that the transmitted intensity decreased with the tilt angle but increased with the field intensity. Miao et al. [70] studied the field intensity sensor achieved by long-period fiber grating. The transmission losses of the resonance wavelength were investigated. For field intensity between 75 Oe and 300 Oe, they reported that the sensitivity of the sensor can reach $0.05 \mathrm{~dB} \mathrm{Oe}^{-1}$ between the field intensity. Recently, D-shaped Bragg grating was employed by Ying et al. [67] to design the ferrofluid-based field intensity sensor as shown in Fig. 27. The ferrofluid film is encapsulated in the above flat surface of the device. The imposed magnetic field can cause the change of refractive index in ferrofluids, thus leading to 
the variation in light transmission of the system. Moreover, they reported that the optimal transmission characteristics occurred for optical fiber sensor with a tilted angle of $8^{\circ}$. For field intensity between 30 Oe and 270 Oe, the wavelength sensitivity was determined to be as high as $-0.18 \mathrm{~nm} \mathrm{Oe}^{-1}$. High sensitivity, good linearity and low cost make their proposed sensor very promising in determining the magnetic field intensity in practice.

\section{Conclusions}

This review covers the recent developments in magneto-optical transmission in magnetic nanofluids for different optical applications which are widely employed in various solar energy conversion processes. The main conclusions and future work are summarized in the following:

- The field-induced aggregation of magnetic nanoparticles, including the chainlike formation and lateral coalescence processes, plays a significant role in determining the transmitted intensity in ferrofluids with magnetic field.

- For light along the field direction, general conclusions were obtained that the time-dependent transmitted intensity first reduced to the minimum when the field is applied and then it will reversely increase along with the time. Besides, beyond the field intensity of certain value, the field-dependent transmitted intensity first increases and follows by decrease with the increasing in field intensity. It is strongly suggested that the combined effect of field intensity and time evolution investigate simultaneously in the future study.

- For light normal to the field direction, the light polarization has great influence on the magneto-optical transmission in ferrofluids. The general conclusions are the transmitted intensity will decrease when $\mathbf{E} / / \mathbf{H}$ while it will increase when $\mathbf{E} \perp \mathbf{H}$. However, there are disagreements among different studies with respect to time evolution of transmitted intensity in the presence of magnetic field. Therefore, further investigations for field-induced time-dependent light transmission in ferrofluids are still needed.

- Due to the magnetic field tunability of optical properties, the ferrofluids can find numerous applications in different fields. Based on the magneto-optical transmission in ferrofluids, various magnetic field fiber sensors were proposed in the past several years. By combining the magnetic nanofluids and different fiber gratings, the sensor can be designed with high sensitivity, good linearity and low cost.

\section{Acknowledgments}

The authors gratefully acknowledge the financial supports of the National Natural Science Foundation of China (No. 51776165). This work was also supported by the China Fundamental Research Funds for the Central Universities. 


\section{References}

[1] Nkurikiyimfura I, Wang Y, Pan Z. Heat transfer enhancement by magnetic nanofluids-A review. Renew Sust Energ Rev. 2013;21:548-61.

[2] Bahiraei M, Hangi M. Flow and heat transfer characteristics of magnetic nanofluids: A review. J Magn Magn Mater. $2015 ; 374: 125-38$

[3] Davies HW, Llewellyn JP. Magnetic birefringence of ferrofluids. II. Pulsed field measurements. J Phys D Appl Phys. $1979 ; 12: 1357$

[4] Yusuf NA, Rousan AA, El-Ghanem HM. Determination of Faraday rotation in a ferrofluid. J Magn Magn Mater. $1987 ; 65: 282-4$

[5] Xu M, Ridler PJ. Linear dichroism and birefringence effects in magnetic fluids. J Appl Phys. 1997;82:326-32.

[6] Li J, Liu XD, Lin YQ, Bai L, Li Q, Chen XM, et al. Field modulation of light transmission through ferrofluid film. Appl Phys Lett. 2007;91:3.

[7] Philip J, Laskar JM. Optical properties and applications of ferrofluids_A review. J Nanofluids. 2012;1:3-20.

[8] Hwang YH, Wu Xl. Quasi-two-dimensional domain structures of magnetic particles in a static field. Phys Rev E. $1994 ; 49: 3102-8$

[9] Liu J, Lawrence EM, Wu A, Ivey ML, Flores GA, Javier K, et al. Field-induced structures in ferrofluid emulsions. Phys Rev Lett. 1995;74:2828-31.

[10] Ivey M, Liu J, Zhu Y, Cutillas S. Magnetic-field-induced structural transitions in a ferrofluid emulsion. Phys Rev E. 2000;63:011403.

[11] Islam MF, Lin KH, Lacoste D, Lubensky TC, Yodh AG. Field-induced structures in miscible ferrofluid suspensions with and without latex spheres. Phys Rev E. 2003;67:021402.

[12] Mehta RV, Patel R, Desai R, Upadhyay RV, Parekh K. Experimental evidence of zero forward scattering by magnetic spheres. Phys Rev Lett. 2006;96:127402.

[13] Philip J, Laskar JM, Raj B. Magnetic field induced extinction of light in a suspension of Fe3O4 nanoparticles. Appl Phys Lett. 2008;92:221911.

[14] Laskar JM, Philip J, Raj B. Light scattering in a magnetically polarizable nanoparticle suspension. Phys Rev E. 2008;78:031404.

[15] Rablau C, Vaishnava P, Sudakar C, Tackett R, Lawes G, Naik R. Magnetic-field-induced optical anisotropy in ferrofluids: A time-dependent light-scattering investigation. Phys Rev E. 2008;78:051502.

[16] Eloi MTA, Santos JL, Morais PC, Bakuzis AF. Field-induced columnar transition of biocompatible magnetic colloids: 
An aging study by magnetotransmissivity. Phys Rev E. 2010;82:021407.

[17] Li J, Lin Y, Liu X, Zhang Q, Miao H, Fu J, et al. A magnetic field-dependent modulation effect tends to stabilize light transmission through binary ferrofluids. Opt Commun. 2012;285:3111-5.

[18] Vales-Pinzón C, Alvarado-Gil JJ, Medina-Esquivel R, Martínez-Torres P. Polarized light transmission in ferrofluids loaded with carbon nanotubes in the presence of a uniform magnetic field. J Magn Magn Mater. 2014;369:114-21.

[19] Shulyma SI, Tanygin BM, Kovalenko VF, Petrychuk MV. Magneto-optical extinction trend inversion in ferrofluids. J Magn Magn Mater. 2016;416:141-9.

[20] Jin JY, Song DX, Geng JF, Jing DW. Time-dependent scattering of incident light of various wavelengths in ferrofluids under external magnetic field. J Magn Magn Mater. 2018;447:124-33.

[21] Lv RQ, Zhao Y, Li H, Hu HF. Theoretical analysis and experimental measurement of birefringence properties in magnetic fluid subjected to magnetic field. IEEE Trans Magn. 2015;51:5.

[22] Laskar JM, Philip J, Raj B. Experimental evidence for reversible zippering of chains in magnetic nanofluids under external magnetic fields. Phys Rev E. 2009;80:8.

[23] Laskar JM, Philip J, Raj B. Experimental investigation of magnetic-field-induced aggregation kinetics in nonaqueous ferrofluids. Phys Rev E. 2010;82:10.

[24] Wiedenmann A, Keiderling U, Habicht K, Russina M, Gahler R. Dynamics of field-induced ordering in magnetic colloids studied by new time-resolved small-angle neutron-scattering techniques. Phys Rev Lett. 2006;97:4.

[25] Li J, Lin Y, Liu X, Wen B, Zhang T, Zhang Q, et al. The modulation of coupling in the relaxation behavior of light transmitted through binary ferrofluids. Opt Commun. 2010;283:1182-7.

[26] Mehta RV. Polarization dependent extinction coefficients of superparamagnetic colloids in transverse and longitudinal configurations of magnetic field. Opt Mater. 2013;35:1436-42.

[27] Wang ZW, Holm C, Muller HW. Molecular dynamics study on the equilibrium magnetization properties and structure of ferrofluids. Phys Rev E. 2002;66:13.

[28] Heinrich D, Goni AR, Smessaert A, Klapp SHL, Cerioni LMC, Osan TM, et al. Dynamics of the field-induced formation of hexagonal zipped-chain superstructures in magnetic colloids. Phys Rev Lett. 2011;106:4.

[29] Dubina SH, Wedgewood LE. A Brownian dynamics study on ferrofluid colloidal dispersions using an iterative constraint method to satisfy Maxwell's equations. Phys Fluids. 2016;28:22.

[30] Halsey TC, Toor W. Structure of electrorheological fluids. Phys Rev Lett. 1990;65:2820-3.

[31] Martin JE, Odinek J, Halsey TC. Evolution of structure in a quiescent electrorheological fluid. Phys Rev Lett. 1992;69:1524-7. 
[32] Martin JE, Anderson RA, Tigges CP. Simulation of the athermal coarsening of composites structured by a uniaxial field. J Chem Phys. 1998;108:3765-87.

[33] Furst EM, Gast AP. Dynamics and lateral interactions of dipolar chains. Phys Rev E. 2000;62:6916-25.

[34] Laskar JM, Philip J, Raj B. Light scattering in a magnetically polarizable nanoparticle suspension. Phys Rev E. 2008;78:9.

[35] Huang Y, Li D, Li F, Zhu Q, Xie Y. Transmitted light relaxation and microstructure evolution of ferrofluids under gradient magnetic fields. Opt Commun. 2015;338:551-9.

[36] Lin L, Li J, Fu J, Lin Y, Liu X. Preparation, magnetization, and microstructure of ionic ferrofluids based on $\gamma$ Fe2O3/Ni2O3 composite nanoparticles. Mater Chem Phys. 2012;134:407-11.

[37] Shulyma S, Tanygin B, Kovalenko V, Petrychuk M. Nanoferrofluid materials: Advanced structure monitoring using optical transmission in a magnetic field. J Nanomater. 2017;2017:7.

[38] Li J, Liu XD, Lin YQ, Qiu XY, Ma XJ, Huang Y. Field-induced transmission of light in ionic ferrofluids of tunable viscosity. J Phys D Appl Phys. 2004;37:3357-60.

[39] Li CL, Cheng CC, Kuo SW, Huang CF, Chen JK. Manipulation of ferrofluids encapsulated in sandwich structures using alternating magnetic field for high contrast in transmittance. Microfluid Nanofluid. 2015;19:1441-53.

[40] Brojabasi S, Mahendran V, Lahiri BB, Philip J. Temperature dependent light transmission in ferrofluids. Opt Commun. $2015 ; 342: 224-9$

[41] Brojabasi S, Lahiri BB, Philip J. External magnetic field dependent light transmission and scattered speckle pattern in a magnetically polarizable oil-in-water nanoemulsion. Physica B. 2014;454:272-8.

[42] Brojabasi S, Mahendran V, Lahiri BB, Philip J. Near infrared light absorption in magnetic nanoemulsion under external magnetic field. Opt Commun. 2014;323:54-60.

[43] Mohapatra DK, Philip J. Investigations on magnetic field induced optical transparency in magnetic nanofluids. Opt Mater. 2018;76:97-105.

[44] Brojabasi S, Muthukumaran T, Laskar JM, Philip J. The effect of suspended Fe3O4 nanoparticle size on magnetooptical properties of ferrofluids. Opt Commun. 2015;336:278-85.

[45] Laskar JM, Brojabasi S, Raj B, Philip J. Comparison of light scattering from self assembled array of nanoparticle chains with cylinders. Opt Commun. 2012;285:1242-7.

[46] Desai R, Upadhyay RV, Mehta RV. Augmentation of chain formation in a magnetic fluid by the addition of halloysite nanotubes. J Phys D Appl Phys. 2014;47:8.

[47] Malynych S, Moroz I. Time dependent magnetically induced variations in optical transmission of magnetite 
nanoparticle aqueous suspension. Cent Eur J Phys. 2012;10:159-65.

[48] Socoliuc V, Popescu LB. Extinction of polarized light in ferrofluids with different magnetic particle concentrations. J Magn Magn Mater. 2012;324:113-23.

[49] Kopčanský P, Macko D, Horváth D, Kašpárková M, Tima T. Study of magneto-optical effects in a damping-oil-based magnetic fluid in the infrared region. J Magn Magn Mater. 1993;122:150-3.

[50] Fang XP, Xuan YM, Li Q. Theoretical investigation of the extinction coefficient of magnetic fluid. J Nanopart Res. $2013 ; 15: 12$.

[51] Fosa G, Bădescu R, Călugăru G, Bădescu V. Measuring the transmittivity of light: A tool for testing the quality of magnetic liquids. Opt Mater. 2006;28:461-5

[52] Pai C, Varma VB, Srinivasan R, Nagarajan R, Ramanujan RV. Control of magnetofluidic laser scattering of aqueous magnetic fluids. IEEE Magn Lett. 2017;8:5.

[53] Mao Y, Liu J, Ge J. Tuning the transmittance of colloidal solution by changing the orientation of Ag nanoplates in ferrofluid. Langmuir. 2012;28:13112-7.

[54] Lv R-q, Zhao Y, Xu N, Li H. Research on the microstructure and transmission characteristics of magnetic fluids film based on the Monte Carlo method. J Magn Magn Mater. 2013;337-338:23-8.

[55] Kushnir SE, Gavrilov AI, Kazin PE, Grigorieva AV, Tretyakov YD, Jansen M. Synthesis of colloidal solutions of SrFe12O19 plate-like nanoparticles featuring extraordinary magnetic-field-dependent optical transmission. J Mater Chem. 2012;22:18893-901.

[56] Philip J, Jaykumar T, Kalyanasundaram P, Rai B. A tunable optical filter. Meas Sci Technol. 2003;14:1289-94.

[57] Pu S, Chen X, Chen L, Liao W, Chen Y, Xia Y. Suppressing the thermal lens effect by magnetic-field-induced mass transfer and phase separation in a magnetic fluid. Appl Phys Lett. 2005;87:021905.

[58] Nair SS, Thomas J, Suchand Sandeep CS, Anantharaman MR, Philip R. An optical limiter based on ferrofluids. Appl Phys Lett. 2008;92:171908.

[59] Chieh JJ, Yang SY, Horng HE, Hong C-Y, Yang HC. Magnetic-fluid optical-fiber modulators via magnetic modulation. Appl Phys Lett. 2007;90:133505.

[60] Pu S, Chen X, Di Z, Xia Y. Relaxation property of the magnetic-fluid-based fiber-optic evanescent field modulator. J Appl Phys. 2007;101:053532.

[61] Horng HE, Chen CS, Fang KL, Yang SY, Chieh JJ, Hong C-Y, et al. Tunable optical switch using magnetic fluids. Appl Phys Lett. 2004;85:5592-4.

[62] Liao W, Chen X, Chen Y, Pu S, Xia Y, Li Q. Tunable optical fiber filters with magnetic fluids. Appl Phys Lett. 
$2005 ; 87: 151122$.

[63] Liu T, Chen X, Di Z, Zhang J, Li X, Chen J. Tunable magneto-optical wavelength filter of long-period fiber grating with magnetic fluids. Appl Phys Lett. 2007;91:121116.

[64] Zhao Y, Liu X, Lv RQ, Zhang YN, Wang Q. Review on optical fiber sensors based on the refractive index tunability of ferrofluid. J Lightwave Technol. 2017;35:3406-12.

[65] Gimenez AJ, Ramirez-Wong DG, Favela-Camacho SE, Sanchez IC, Yáñez-Limón JM, Luna-Bárcenas G. Optical detection of magnetic nanoparticles in colloidal suspensions. J Magn Magn Mater. 2016;402:150-5.

[66] Yang X, Liu Y, Zheng Y, Li S, Yuan L, Yuan T, et al. A capillary optical fiber modulator derivates from magnetic fluid. Opt Commun. 2013;304:83-6.

[67] Ying Y, Zhang R, Si G-Y, Wang X, Qi Y-W. D-shaped tilted fiber Bragg grating using magnetic fluid for magnetic field sensor. Opt Commun. 2017;405:228-32.

[68] Lin W, Miao Y, Zhang H, Liu B, Liu Y, Song B, et al. Two-dimensional magnetic field vector sensor based on tilted fiber Bragg grating and magnetic fluid. J Lightwave Technol. 2013;31:2599-605.

[69] Yang D, Du L, Xu Z, Jiang Y, Xu J, Wang M, et al. Magnetic field sensing based on tilted fiber Bragg grating coated with nanoparticle magnetic fluid. Appl Phys Lett. 2014;104:061903.

[70] Miao YP, Ma XX, Lin JC, Song BB, Lin W, Zhang H, et al. Intensity-based magnetic field measurement employing tilted long-period fiber gratings. J Opt. 2015;17:5. 